\title{
农业机械轻量化技术研究现状与发展趋势*
}

\author{
才 胜 $^{1}$ 罗颖辉 $^{1}$ 李青林 $^{2}$ \\ (1. 中国农业大学工学院 北京 100083;
}

2. 江苏大学农业装备工程学院 镇江 212013)

\begin{abstract}
摘要: 轻量化是未来车辆发展的重要方向, 也是提升农业装备质量的必由之路。轻量化可以从结构优化、使用轻质材料、采 用先进制造工艺三个方面出发, 在不降低车辆性能和显著提高生产制造成本的前提下, 寻求车辆的最佳结构和最优材料布局。 对近些年来车辆轻量化技术的研究现状和进展以及在农机中的应用进行了综述, 内容主要包括结构轻量化(尺寸优化、形状优 化、拓扑优化和多学科设计优化)、材料轻量化(高强度钢、铝合金、镁合金、钛合金及塑料和复合材料)、工艺轻量化(激光焊 接、热冲压成形、液压成形和铸造成形), 最后对未来轻量化技术的发展方向和趋势进行讨论和探究, 旨在为提高农机轻量化 技术提供参考和借鉴。
\end{abstract}

关键词: 轻量化技术; 轻量化材料; 结构优化; 农业机械

中图分类号: S220

\section{State of the Art of Lightweight Technology in Agricultural Machinery and Its Development Trend}

\author{
CAI Sheng ${ }^{1} \quad$ LUO Yinghui $^{1} \quad$ LI Qinglin ${ }^{2}$ \\ (1. College of Engineering, China Agricultural University, Beijing 100083; \\ 2. School of Agricultural Equipment Engineering, Jiangsu University, Zhenjiang 212013)
}

\begin{abstract}
Lightweight is an important development direction for future vehicle and is of significance for the improvement of agricultural machinery. Lightweight can be realized from three aspects like structural optimization, lightweight materials and advanced manufacturing processes to achieve the optimal structure and optimal material layout of the vehicles under the premise of no reducing the performance of the vehicle and significantly increasing the manufacturing cost. The research status and progress of vehicle lightweight technology and its application in agricultural machinery in recent years are reviewed, mainly including structural lightweight (size optimization, shape optimization, topology optimization and multidisciplinary design optimization), lightweight materials (high-strength steel, aluminum alloy, magnesium alloy, titanium alloy and plastics and composite materials), lightweight process (laser welding, hot stamping, hydro-forming and casting). Finally, the development direction and trend of future lightweight technology are discussed. The aim of this work is to improve the reference for the lightweight technology of future agricultural machinery.
\end{abstract}

Key words: lightweight technology; lightweight materials; structural optimization; agricultural machinery

\section{0 前言}

近些年来，随着汽车工业飞速发展以及农业机 械化水平持续提高, 汽车和非道路车辆成为了世界

* 江苏省现代农业装备与技术协同创新中心资助项目(310)。20210218 收 到初稿, 20210430 收到修改稿
能源消耗和污染物排放的主要来源。随之而来的全 球气候变化、环境污染以及能源危机问题也日益突 出, 节能减排成为全球工业和农业机械工业可持续 发展的一个必然趋势 ${ }^{[1]}$ 。研究表明 ${ }^{[2]}$, 轻量化能有效 地降低油耗, 减少排放。若整车质量减少 $100 \mathrm{~kg}$, 百公里油耗可减少约 $0.3 \sim 0.5 \mathrm{~L}, \mathrm{CO}_{2}$ 排放量相应减 少约 $1 \mathrm{~kg}$ 。“轻量化” 不是单纯地将车辆自身质量 
“减轻”, 而是在保证车辆的强度和各项安全性能前 提下, 最大限度地降低车辆自重, 从而提高车辆的 动力性, 降低燃油消耗, 减少尾气排放 ${ }^{[3]}$ 。

\section{1 轻量化技术的分类}

轻量化技术包括 “结构轻量化、材料轻量化、 工艺轻量化”三个方面, 其中结构轻量化主要包括 尺寸优化、形状优化、拓扑优化和多学科设计优化 (MDO); 材料轻量化主要包括高强度钢、铝合金、 镁合金、钛合金、塑料和复合材料; 工艺轻量化主 要包括激光焊接、热冲压成形、液压成形和铸造成 形等, 如图 1 所示 ${ }^{[4]}$ 。

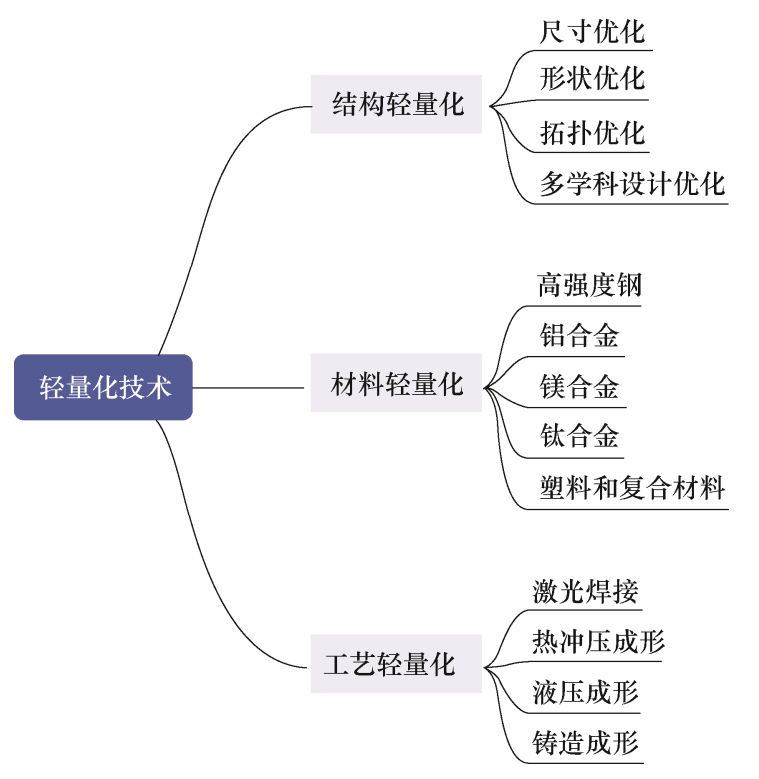

图 1 轻量化技术分类

\section{2 结构轻量化}

\section{1 尺寸优化}

尺寸优化是结构优化设计中应用最广泛最成熟 的优化设计方法 ${ }^{[5]}$, 它是指对优化设计对象的截面 尺寸、壁厚、减重孔(槽)尺寸等尺寸参数进行合理 改进以达到轻量化的目的。左文杰等人 ${ }^{[6]}$ 基于尺寸 优化方法对某轿车的白车身进行轻量化设计, 采用 序列线性规划法求解所建立的数学优化模型, 结果 显示, 优化后的车身质量减轻 $34.6 \mathrm{~kg}$, 减重率达 $11.4 \%$ 。于志新等 ${ }^{[7]}$ 利用 Hyperworks 软件对某载货 汽车车架进行优化设计, 基于满载弯曲、满载扭转、 紧急制动三种工况仿真分析结果, 对车架的 2 根纵 梁和 8 根横梁的厚度进行了优化改进(约束其质量不 超过 $970 \mathrm{~kg}$ ), 其中 2 根纵梁减薄 $2.6 \mathrm{~mm}, 4$ 根横梁
分别减薄 $3.4 \mathrm{~mm} 、 3.7 \mathrm{~mm} 、 3.9 \mathrm{~mm} 、 3.6 \mathrm{~mm}$, 另外 4 根横梁分别增厚 $9.8 \mathrm{~mm} 、 10.1 \mathrm{~mm} 、 10.3 \mathrm{~mm}$ 、

$9 \mathrm{~mm}$, 优化后车架的最大位移由原来的 $20.0 \mathrm{~mm}$ 减 至 $12.3 \mathrm{~mm}$, 刚度提高了 $8 \%$ 。宋晓飞等 ${ }^{[8]}$ 基于 Optistruct 软件采用尺寸优化(桥壳减薄 $2 \mathrm{~mm}$, 板簧 座减薄 $2 \mathrm{~mm}$, 后盖减薄 $1 \mathrm{~mm}$, 轴管减薄 $1 \mathrm{~mm}$, 加强圈减薄 $5 \mathrm{~mm}$ ) 对某重型汽车的驱动桥壳进行轻 量化设计, 在满足桥壳强度、刚度、模态及疲劳寿 命等性能要求的前提下, 实现对桥壳减重 $50.075 \mathrm{~kg}$, 减重率达 $24.78 \%$ 。黄纬等 ${ }^{[9]}$ 从截面优化 和厚度优化两个方面对某汽车后悬架摆臂进行尺寸 优化(主体下板减薄 $1 \mathrm{~mm}$, 加强上板减薄 $1 \mathrm{~mm}$, 弹 簧板座增厚 $1 \mathrm{~mm}$ ), 最终实现了摆臂减重 $23 \%$, 同 时提高了摆臂的强度, 并且通过仿真和试验验证了 优化方案的可行性。王震虎等 ${ }^{[10]}$ 基于真实接头的白 车身概念模型, 采用序列二次规划法对通过灵敏度 分析篮选出 19 个主断面进行尺寸优化设计, 最终实 现了对概念车身的 $8.33 \mathrm{~kg}$ 减重, 减重率达 $2.7 \%$ 。 周琪等 ${ }^{[11]}$ 基于碳纤维缠绕内衬钢管方案对某承载式 车架进行轻量化设计, 经自由尺寸优化、厚度参数 优化、铺层顺序优化得到了最佳 CFRP 铺层方案, 结果表明, 优化后的车架较原钢制车架质量减轻 $7.62 \mathrm{~kg}$, 减重率达 $23 \%$ 。LI 等 ${ }^{[12]}$ 以某全铝白车身为 研究对象, 基于刚度和一阶模态灵敏度分析篮选出 了 20 个位置的车身厚度作为优化设计变量, 结合结 构改进使用尺寸优化方法对白车身进行轻量化设 计, 结果显示, 优化后的白车身较优化前减重 $28 \mathrm{~kg}$, 减重率达 9.2\%, 且弯曲刚度和模态性能较优化前都 有一定程度的提高。HOU 等 ${ }^{[13]}$ 对某帽形汽车 $\mathrm{T}$ 型 接头的尺寸和形状进行了优化改进, 优化后的结构 较优化前质量减轻 $86.5 \mathrm{~g}$, 减重率达 $10 \%$, 且弯曲 刚度得到了显著提升。姜丽红等 ${ }^{[14]}$ 基于模态和强度 分析结果, 使用尺寸优化方法(上板减薄 $0.5 \mathrm{~mm}$, 下板减薄 $0.2 \mathrm{~mm}$, 连接板减薄 $0.5 \mathrm{~mm}$ ) 对某汽车前 副车架进行轻量化设计, 实现车架减重 $1.9 \mathrm{~kg}$, 减重 率达 $9.9 \%$, 且通过实车试验和仿真分析验证了优化 方案的可行性。

\section{2 形状优化}

形状优化指在保证结构的拓扑关系不变的情况 下, 调整优化结构设计区域的形状和内边界尺寸, 找到结构的最优几何形状。谷正气等 ${ }^{[15]}$ 基于 Kriging 模型对某导流罩的形状进行了优化设计(使用多岛 遗传算法对近似模型进行优化求解), 最终使集装箱 半挂车的阻力系数降低了 $9.73 \%$ 。廖莺等 ${ }^{[16]}$ 在概念 设计阶段对某铝合金副车架进行轻量化设计，通过 
竞品分析确定了车架的质量、模态、刚度和强度等 目标, 利用 ICM 混合建模方法建立了优化模型, 通 过拓扑优化和形状优化两级优化实现了对车架的 $1.84 \mathrm{~kg}$ 减重。

形貌优化作为形状优化的高级形式, 它是指在 板形结构中寻找最优的加强肋分布概念设计方法。 陈静等 ${ }^{[17]}$ 在对某电动汽车的电池箱进行轻量化设 计时, 采用形貌优化和尺寸优化 (以质量最小为优化 目标函数, 以上箱体厚度为设计变量, 以第一阶模 态频率高于 $30 \mathrm{~Hz}$ 为约束条件, 建立优化模型)对电 池箱的上箱体进行优化设计, 基于碳纤维复合材料 替换原铝合金材料的优化方案, 采用自由尺寸优化、 尺寸优化、多目标优化、顺序优化方法对碳纤维复 合材料下箱体进行铺层厚度和铺层顺序优化, 最终 使得电池箱的质量减轻 $29.8 \%$ 。陆善涁等 ${ }^{[18]}$ 利用等 效静态载荷方法(ELSM)对某汽车的保险杜、吸能盒 和前纵梁 3 个主要前端结构组件的尺寸和形状进行 了耐撞性优化设计, 优化结果显示, 优化后的结构 相比优化前轻 $7.03 \%$, 且碰撞性能较优化前也有了 一定程度的提升。

\section{3 拓扑优化}

拓扑优化是指在给定的设计空间内, 以其材料 分布为优化目标函数, 通过优化算法得到最佳分布 方案和最佳传力路径以使结构的某项性能指标达到 最佳。相比于尺寸优化和形状优化, 拓扑优化具有 更大的优化设计空间, 是结构优化中最具发展前景 的一种方法。拓扑优化可以分为连续体拓扑优化和 离散结构拓扑优化。连续体拓扑优化方法是基于有 限元方法将优化设计区间的材料离散成有限个单元 (壳单元或体单元), 而离散结构拓扑优化是在优化 设计区间内建立一个由有限个梁单元组成的基结 构, 然后通过优化算法确定设计区间内单元的去留, 所有保留下来的单元构成最终的拓扑优化方案, 从 而完成拓扑优化设计。目前, 在众多拓扑优化方法 中, 使用最为广泛的是变密度法连续体拓扑优化。 张楠等人 ${ }^{[19]}$ 采用拓扑优化方法对某变速箱箱体进 行轻量化设计, 以变速箱箱体质量最小为优化目标 函数, 以箱体的应力和相关位置位移为约束条件, 建立拓扑优化数学模型, 结果显示, 优化后的箱体 减轻 $1.9 \mathrm{~kg}$, 减重率达 $9.98 \%$ 。刘洋等 ${ }^{[20]}$ 基于 Inspire 软件对某发动机附件的复合支架进行轻量化设计, 采用拓扑优化方法实现对支架的 $60 \%$ 的减重, 并且一 定程度地提高了支架的模态和强度性能。李雨萱 ${ }^{[21]}$ 采用拓扑优化方法对某重型汽车平衡悬架进行轻量 化设计, 以平衡轴质量最小为优化目标函数, 以模
态、应力和变形为约束条件建立拓扑优化模型, 实 现了对平衡轴座的 $5.9 \mathrm{~kg}$ 减重, 减重率达 $7.6 \%$ 。周 思等 ${ }^{[22]}$ 基于铝合金代替铸铁方案，使用拓扑优化方 法对某汽车转向节进行优化设计, 使得转向节成功 减重 $2.05 \mathrm{~kg}$, 减重率高达 $48 \%$ 。VIQARUDDIN 等 人 ${ }^{[23]}$ 采用拓扑优化方法对某铝合金制汽车控制臂 进行了结构优化设计, 实现了控制臂 $8.2 \mathrm{~kg}$ 减重, 减重率达 $41 \%$, 且优化后的控制臂满足强度和刚度 要求。JUNG 等 ${ }^{[24]}$ 基于钢铝材料混合方案对某电动 客车的车顶结构进行了多材料拓扑优化设计, 优化 后的钢铝混合车顶较原铝合金车顶质量减轻 $8.0 \%$ 。

\section{4 多学科设计优化}

传统只考虑单一学科性能要求的优化设计方法 不能满足现代车身结构设计要求, 为了得到满足汽 车强度、模态、NVH 性能、耐撞性等性能的最优解 集, 多学科设计优化方法在汽车轻量化设计中得到 广泛使用。多学科设计优化的数学模型一般表达 式 ${ }^{[25]}$ 为

$$
\begin{aligned}
\min & f(x, y(x)) \\
\text { s. t. } & h_{i}(x, y(x))=0, \quad i=1,2, \cdots, m \\
& g_{j}(x, y(x)) \leqslant 0, \quad j=1,2, \cdots, n
\end{aligned}
$$

式中, $f(x, y(x))$ 为优化目标函数; $h_{i}(x, y(x))$ 、 $g_{j}(x, y(x))$ 为约束条件, $x$ 为优化设计变量; $y(x)$ 为 系统分析方程 $A(x, y(x)$ ) 确定的状态方程。

系统分析方程 $A(x, y(x))$ 为

$$
A(x, y(x))=\left(\begin{array}{c}
A_{1}\left(x, y_{1}(x), \cdots, y_{N}(x)\right) \\
\vdots \\
A_{N}\left(x, y_{1}(x), \cdots, y_{N}(x)\right)
\end{array}\right)=0
$$

式中, $N$ 为 $\mathrm{MDO}$ 子系统的数目。

史朝军等 ${ }^{[26]}$ 运用多学科优化设计方法对某自 卸车货箱进行轻量化设计, 在保证自卸车货箱的刚 度、强度、第一阶模态频率不降低的基础上, 实现 了货箱减重 $230 \mathrm{~kg}$, 减重率达 $8.3 \%$ 。史国宏等 ${ }^{[27]}$ 根据白车身自身性能特点将车身分成 3 个优化设计 空间, 对由试验设计采用 Kriging 方法建立的近似 模型进行多学科优化设计, 分别实现对三个区域减 重 $6 \mathrm{~kg} 、 1 \mathrm{~kg} 、 5 \mathrm{~kg}$, 整个白车身减重 $12 \mathrm{~kg}$, 减重 率达 $4.5 \%$ 。成艾国等 ${ }^{[28]}$ 运用最优拉丁试验设计方法 和移动最小二乘法建立了整车正碰、白车身刚度以 及强度多学科的响应面模型, 在满足正碰法规、白 车身强度和刚度要求的基础上, 通过采用热成形车 架, 取得了较好的减重效果。刘钊等 ${ }^{[29]}$ 结合加强协 同多学科优化理论, 基于粒子群算法对某商用车车 身的侧面结构进行轻量化设计研究, 实现了对侧面 
结构的最多减重 $12.6 \mathrm{~kg}$, 减重率达 $14.6 \%$ 。于成龙 等 ${ }^{[30]}$ 运用多学科优化设计方法对某电动车的锂离 子动力电池包进行轻量化设计 (建立了电池包的 $\mathrm{NVH}$ 、结构耐久和碰撞安全的多学科优化模型), 在 满足电池包各项性能要求的基础上, 成功实现了对 电池包的 $4 \mathrm{~kg}$ 减重。高大威等 ${ }^{[31]}$ 以超高强度钢 $22 \mathrm{MnB} 5$ 代替原材料(DC01)对某款轻型面包车车门 防撞杆进行了多学科优化设计, 在不降低防撞杆性 能的基础上, 实现对防撞杆减重 $34.7 \%$, 轻量化效 果显著。XIONG 等 ${ }^{[2]}$ 综合考虑模态、刚度、耐撞性 能对某汽车前端结构进行了结构一材料一体化轻量 化设计 (对 27 个关键位置的尺寸和材料类型进行优 化), 实现了车身减重 $4.12 \mathrm{~kg}$, 减重率达 $1.32 \%$, 同 时也降低了材料成本。MIAO 等 ${ }^{[33]}$ 基于疲劳评估对 某火车车厢进行了多学科优化设计, 实现了车厢减 重 $538.75 \mathrm{~kg}$, 减重率达 $6.68 \%$ 。

\section{3 材料轻量化}

\section{1 高强度钢材料}

高强度钢在汽车轻量化中发挥着非常重要的作 用。用高强度钢板取代普通钢板, 在保证其使用性 能的同时可以减小板材厚度, 从而减轻汽车质量。 目前汽车用高强度钢主要有双相钢(DP 钢)、复相钢 (CP 钢)、相变诱导塑性钢(TRIP 钢)、马氏体钢(MS 钢)、淬火延性钢( $\mathrm{QP}$ 钢)、孪晶诱发塑性钢(TWIP 钢)、硼钢(B 钢)。为了满足轻量化、节能减排、高 强度和长疲劳寿命的需求, 首钢集团研发了一系列 轻质高强度桥壳用钢, 包括热冲压成形钢、冷冲压 成形钢和机械扩胀成形钢, 见表 $1^{[34]}$ 。高新华等 ${ }^{[35]}$ 采用高强度钢(1000DP)对某款轿车的门槛梁进行轻 量化设计, 首先使用尺寸优化方法在保证门槛梁吸 能特性不降低的情况下减重 $13.6 \%$, 进而针对高强

表 1 首钢轻质桥壳用钢简介

\begin{tabular}{llll}
\hline 成形工艺 & 牌号 & 厚度/mm & 研发进程 \\
\hline \multirow{6}{*}{ 热冲压 } & SQ420RQK & $3.0 \sim 20.0$ & 稳定批量生产 \\
& SQ460RQK & $3.0 \sim 20.0$ & 稳定批量生产 \\
& SQ500RQK & $3.0 \sim 20.0$ & 稳定批量生产 \\
& SQ550RQK & $3.0 \sim 20.0$ & 小批量生产 \\
& SQ420LQK & $3.0 \sim 18.0$ & 稳定批量生产 \\
冷冲压 & SQ460LQK & $3.0 \sim 16.0$ & 稳定批量生产 \\
& SQ500LQK & $3.0 \sim 14.0$ & 稳定批量生产 \\
& SQ550LQK & $3.0 \sim 14.0$ & 稳定批量生产 \\
& SQ600LQK & $3.0 \sim 14.0$ & 小批量生产 \\
& SQK600ZX & $4.0 \sim 14.0$ & 稳定批量生产 \\
机械扩胀 & SQK650ZX & $4.0 ~ 14.0$ & 稳定批量生产 \\
& SQK700ZX & $4.0 \sim 14.0$ & 稳定批量生产 \\
& SQK750ZX & $4.0 \sim 14.0$ & 稳定批量生产 \\
\hline
\end{tabular}

注: 牌号中 $\mathrm{S}$ 代表首钢, $\mathrm{Q}$ 代表屈服强度, $\mathrm{R}$ 代表热冲压, $\mathrm{L}$ 代表冷 冲压, QK 代表桥壳, ZX 代表胀形。
度钢的开裂和回弹等问题, 改变了压边力、合理布 置了拉延筋以及对门槛梁局部结构形状进行了改 进, 最后, 对门槛梁内板和加强板的厚度进行了二 次优化, 使得门槛梁质量减轻 $16 \%$ 并且提高了门槛 梁的吸能效率。

周森 ${ }^{[36]}$ 使用 $715 \mathrm{MPa}$ 级高强度调质钢材料设计 了一液化石油气运输半挂车罐体, 该罐体壁厚相比 于原同款车型的罐体减薄 $1 \mathrm{~mm}$, 质量减轻约 $700 \mathrm{~kg}$, 且搭载该罐体的样车通过了国家专业车辆 鉴定部门的鉴定。

\section{2 铝合金材料}

铝合金的密度约为钢的密度的 $1 / 3$, 比强度接近 高合金钢，比刚度大，并且具有良好的耐腐蚀性、 导电性、导热性、成形性等特点 ${ }^{[37]}$, 是汽车轻量化 的理想材料之一。特斯拉 Model S 采用了全铝合金 车身有效地减轻了车身自重, 蔚来 ES8 不仅采取全 铝车身, 而且它的前、后副车架、底盘以及悬挂系 统均采用铝合金材料, 整车用铝率高达 $96 \%$, 大大 的减轻了汽车自重, 2016 年, 本田展示了其运用铝 压铸法加工而成的全新铝合金副车架，该副车架平 均壁厚约为 $3 \mathrm{~mm}$, 比原来的副车架轻 $20 \%$ 。奇瑞 汽车等公司联合开发了一系列铝合金底盘零部件, 见表 $2^{[38]}$ 。林佳武等 ${ }^{[39]}$ 采用真空高压铸造铝合金代 替传统钢对某电动车后纵梁进行轻量化设计, 综合 考虑后撞耐撞性、压铸成形、连接工艺等要求设计 了压铸铝合金后纵梁, 最后对产品性能进行了分析, 验证了产品的可行性, 实现了后纵梁质量减轻 9.3 $\mathrm{kg}$, 减重率达 $31 \%$ 。王俊峰等 ${ }^{[40]}$ 在对某车型发动机 舱盖进行轻量化设计时, 采用铝合金(AA5182)材料 替换传统钢制的发动机内、外板, 实现了发动机舱 盖 $7.70 \mathrm{~kg}$ 减重, 减重率高达 $50.8 \%$ 。东风传动轴公 司成功研制了铝合金传动轴, 相比于传统钢制传动 轴, 质量减轻 $12 \mathrm{~kg}$, 减重率达 $30 \%$, 并且采用铝合 金传动轴能提高整车的 NVH 性能。金泉军等人 ${ }^{[41]}$ 使用 6082-T6、6063-T6 铝合金材料替换传统的双相 钢(HC550/980DP) 材料对汽车防撞梁进行轻量化设 计, 最终在满足防撞梁模态、刚度、碰撞性能等要 求下, 实现了对防撞梁的 $3.35 \mathrm{~kg}$ 减重, 减重率高达 $50.7 \%$ 。刘雅芳等 ${ }^{[42]}$ 基于 LS-DYNA 软件对国内某轿 车车门防撞杆进行轻量化设计时, 将钢材料替换成 6082 铝合金材料, 实现了防撞杆的 $1.317 \mathrm{~kg}$ 减重, 减重率高达 $48.9 \%$, 此外, 还提高了车门的碰撞吸 能特性。张琼等 ${ }^{[43]}$ 对某纯电动客车车身骨架以铝合 金材料代替高强度钢进行轻量设计研究, 结果显示, 铝合金轻量化后的车身骨架不仅满足刚度、模态等 
要求, 并且比高强度钢车身骨架轻 $195 \mathrm{~kg}$ 。

表 2 铝合金底盘零部件

\begin{tabular}{ccccc}
\hline & $\begin{array}{c}\text { 钢制件 } \\
\text { 质量 } / \mathrm{kg}\end{array}$ & $\begin{array}{c}\text { 铝制件 } \\
\text { 质量 } / \mathrm{kg}\end{array}$ & $\begin{array}{c}\text { 轻量化效 } \\
\text { 果 }(\%)\end{array}$ & $\begin{array}{c}\text { 减重 } \\
\text { 质量 } / \mathrm{kg}\end{array}$ \\
\hline $\begin{array}{c}\text { 转向节 } \\
\text { 前副车 }\end{array}$ & 4.4 & 1.8 & 59.1 & 2.6 \\
$\begin{array}{c}\text { 架 } \\
\text { 后副车 }\end{array}$ & 24.6 & 13.5 & 45.1 & 11.1 \\
$\begin{array}{c}\text { 架 } \\
\text { 控制臂 }\end{array}$ & 20 & 11.5 & 42.5 & 8.5 \\
\hline
\end{tabular}

\section{3 镁合金材料}

镁合金是一种比铝合金更轻的材料, 其密度大 约为铝的密度的三分之二, 是目前工业用金属材料 中最轻的合金材料。镁合金具有质量轻、抗震、抗 干扰、良好的耐蚀性、熔点低、良好的阻尼性等特 点, 在汽车内饰(转向盘、仪表盘横梁、座椅)、动 力系统(曲轴箱零件、凸轮盖、气缸盖罩、进气歧管、 阀套等)、底盘系统(镁合金轮㪍、悬架控制臂、发 动机支架、散热器支架、转向节等)、车身(车身板 材、骨架、车门内框、车顶框架等)中都有使用 ${ }^{[44-45]}$ 。 上海汽车集团有限公司技术中心的陈甜斌 ${ }^{[46]}$ 设计 了一款镁合金座椅骨架, 其中, 镁合金靠背比原钢 制的靠背轻 $1.14 \mathrm{~kg}$, 镁合金座盆比原钢制座盆轻 $0.94 \mathrm{~kg}$, 减重率分别为 $44.5 \%, 37.2 \%$, 减重效果显 著, 并且通过仿真分析验证了该座椅骨架的可靠性。 刘杰等 ${ }^{[47]}$ 探究了锻压工艺参数(始锻温度、终锻温度 和锻压比)对镁合金( $\mathrm{Mg}-8 \mathrm{Al}-0.6 \mathrm{Zn}-0.3 \mathrm{Ce})$ 车轮耐蚀 性性能的影响, 最终得到车轮的最佳铅压工艺参数 为: 始锻温度 $360{ }^{\circ} \mathrm{C}$ 、终锻温度 $260{ }^{\circ} \mathrm{C}$ 、锻压比 14。LÜ 等 ${ }^{[48]}$ 基于镁合金材料对某汽车底盘车架进 行轻量化设计, 仿真结果显示, 在车架梁截面形状 一致情况下，使用镁合金代替铝合金或钢可以显著 减轻车架质量, 且不影响车架的强度和刚度性能。 高景峰等 ${ }^{[49]}$ 用差压铸造法制备了汽车零件用镁合 金 Mg-9Al-1Zn-0.5Ca, 并且对其组织、力学性能和 耐磨损性能进行了测试和分析, 结果显示, 差压铸 造可以明显地改善合金的内部组织并提高合金的力 学性能和耐磨性性能。

\section{4 钛合金材料}

钛合金的密度大约为钢的三分之二, 具有比强 度高、塑性好、耐高温、耐疲劳等优点, 但因其制 造成本高、加工工艺要求高, 在汽车领域未得到广 泛的应用, 主要应用于部分高端高性能汽车的发动 机连杆轴、凸轮轴、气门弹簧座、涡轮增压器等零 部件中 ${ }^{[50]}$ 。2014 年 12 月 17 日本新日铁住公司发布 消息称, 雅马哈发动机公司的新型竞技摩托车
“YZFR1”、“YZF-R1M” 的发动机连杆采用了公司 生产的钛合金 ${ }^{[51]}$ 。2017 年, 德国大众豪华汽车品牌 布加迪推出了世界上第一款 3D 打印制造的钛合金 制动钳, 这款制动钳是全球汽车行业最大的 $3 \mathrm{D}$ 打 印钛合金零部件 ${ }^{[52]}$ 。

\section{5 塑料和复合材料}

塑料和复合材料具有质量轻、性能优良、功能 性强等特点 ${ }^{[53]}$, 是汽车轻量化材料的一个重点关注 领域。近几年, 塑料和复合材料在汽车中的使用量 有了快速的提高, 目前汽车用塑料以聚丙烯( $\mathrm{PP}$ )、 尼龙 $(\mathrm{PA})$ 、丙烯腈一丁二烯一苯乙烯共聚物( $\mathrm{ABS})$ 、 聚碳酸酯 $(\mathrm{PC})$ 、聚乙烯 $(\mathrm{PE})$ 、聚氯乙烯 $(\mathrm{PVC})$ 和聚甲 醛 $(\mathrm{POM})$ 等为主, 广泛应用于汽车内饰件、车身覆 盖件等汽车零部件中; 汽车用复合材料以 PP、PA6 和 PA66 等为主, 主要应用于汽车前端结构、电动 汽车电池包等零部件中 ${ }^{[54]}$ 。陈静等 ${ }^{[55]}$ 为某车型设计 了一款碳纤维复合材料防撞梁, 采用全因子实验设 计确定了防撞梁的最优截面形状和铺层顺序组合, 最后使用遗传算法对防撞梁结构铺层厚度进行了多 目标优化, 得到的最优碳纤维复合材料防撞梁质量 仅为 $0.825 \mathrm{~kg}$, 相比于钢制防撞梁轻 $1.496 \mathrm{~kg}$, 减重 率高达 $64.5 \%$ 。王庆等 ${ }^{[56]}$ 为某款电动汽车设计了一 整体式 CFRP 复合材料保险杜, 进而对保险杜进行 轻量化设计, 使用拉丁超立方采样和 Kriging 近似 模型技术建立了优化近似模型, 使用遗传算法求解 得到了优化结果, 结果显示, 优化设计方案较原铝 合金结构轻 $0.91 \mathrm{~kg}$, 减重率达 $36.4 \%$, 且通过仿真 分析验证了优化设计方案的可靠性。LIU 等 ${ }^{[57]}$ 基于 改进粒子群算法使用碳纤维增强复合材料代替铝合 金材料对某商用车保险杜进行了轻量化设计研究, 综合考虑拖钩强度、低速碰撞性能和 NVH 性能对 保险杜的 9 个关键尺寸参数进行了优化设计, 优化 结果显示, 轻量化设计方案较原铝合金结构质量减 轻 $0.837 \mathrm{~kg}$, 减重率达 $31.5 \%$, 且通过实车试验验证 了方案的强度可靠性和耐撞性。WEI 等 ${ }^{[58]}$ 基于改进 粒子群算法对某三维编织复合材料挡泥板进行了多 尺度优化设计, 在满足多种工况下刚度要求的基础 上, 实现挡泥板的 $0.43 \mathrm{~kg}$ 减重, 减重率达 $20.65 \%$ 。

\section{4 工艺轻量化}

\section{1 激光焊接}

激光焊接因具有能量密度高、无接触、焊接效 率高、焊接速度快等优点, 在汽车、船舶、航空航 天等制造业领域得到了广泛的应用 ${ }^{[59]}$ ，其中激光拼 
焊在汽车制造中应用较为成熟。宝马 7 系轿车的铝 合金车门采用了激光焊接技术，相比于传统的钢制 车门质量减轻 $30 \%$ 左右。黄林等 ${ }^{[60]}$ 从成形性、生产 成本和效率等方面对比分析了激光拼焊全景天窗加 强框和传统焊接全景天窗加强框, 分析结果表明, 相比于传统焊接加强框, 激光拼焊加强框总质量减 少 $1.17 \mathrm{~kg}$, 并且在成形性和生产成本方面更具优势。 王登峰等 ${ }^{[61}$ 基于激光拼焊技术，综合使用最优拉丁 超立方采样技术、径向基近似模型理论和非支配遗 传算法对某汽车 B 柱进行多目标轻量化优化设计, 获得了 Pareto 最优解集, 按需选取了其中两种妥协 方案验证了近似模型的可靠性和优化设计效果, 其 中一种方案表明 B 柱质量较优化前减轻 $18.24 \%$, 同 时提高了汽车的侧面碰撞安全性能。SUN 等 ${ }^{[62]}$ 基于 多材料激光拼焊板技术对某汽车车门进行了多目标 多材料优化设计, 结果显示, 优化后的车门较优化 前质量减轻 $4.1 \mathrm{~kg}$, 减重率达 $42.1 \%$, 且模态和刚度 等性能较优化前有较大的改善。

\section{2 热冲压成形}

针对常温下高强度钢板变形范围小、易开裂、 回弹值大等 ${ }^{[63]}$ 问题, 热冲压成形技术应运而生, 它 是一项专门用于成形高强度钢板的成形技术。国外 方面, 像沃尔沃、保时捷、大众等公司都采用了超 高强度钢热冲压成形技术制造车身零部件, 国内方 面, 如宝钢 ${ }^{[64]}$ 集团为某用户提供的 B 柱进行改型设 计, 将冷冲压 $\mathrm{B}$ 柱改型为热冲压 $\mathrm{B}$ 柱, 最后相比于 冷冲压 B 柱, 热冲压 B 柱质量减轻 35.7\%, 并且其 强度和刚度得到了显著的提高。

\section{3 液压成形}

液压成形技术作为一种轻量化先进制造技术在汽 车工业中的应用越来越多。相比于传统冲压焊接件, 液压成形件具有生产成本低、强度和刚度大、质量轻 等优点 ${ }^{[65]}$ 。目前, 汽车上的液压成形件包括副车架、 保险杜、控制臂、转向杆、进排气歧管、前壁板横梁 等。图 $2^{[66-67]}$ 为典型的汽车液压成形零部件。

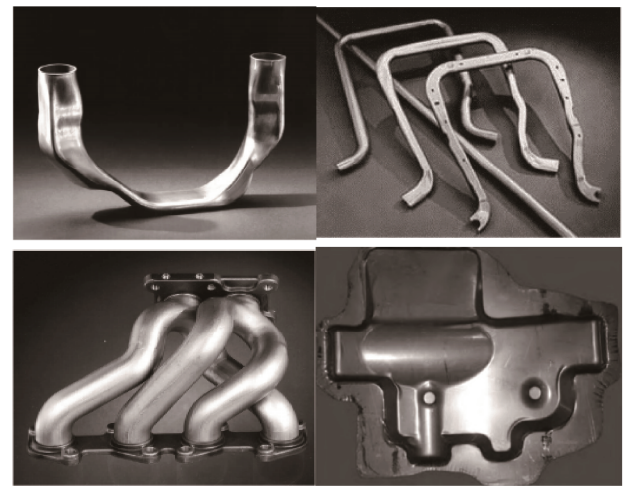

图 2 典型液压成形结构件
陈仙风等 ${ }^{[68]}$ 以某轿车前保险杜吸能盒为研究 对象, 研制了液压成形管件替代传统的冲压焊接结 构, 成功减重 $10.2 \%$, 并且通过试验验证了液压成 形吸能盒相比于冲压焊接结构在吸能方面更有优 势。徐鸣涛等 ${ }^{[69]}$ 基于管件液压成形技术对某 SUV 的吸能盒进行结构改进, 结果表明, 液压成形吸能 盒较原冲压吸能盒质量减轻 $0.065 \mathrm{~kg}$, 且吸能特性 更优。

\section{4 铸造成形}

在汽车制造业中, 铸造是一种制造轻量化镁铝 汽车零部件的高效工艺, 它主要包括真空铸造、真 空辅助压铸、高真空压铸、低压铸造等。将传统的 冲压、焊接成形等几个零件, 通过铸造成一个集成 零件可以达到显著的减重效果, 使用铸造成形的轻 质材料能使得轻量化效果得到进一步提高。别克君 悦车型采用了压铸镁仪表板梁, 该板梁质量仅为 $6.9 \mathrm{~kg}$ ，相比于传统的的钢制仪表板梁，减重 $40 \%$, 如图 $3^{[70]}$ 所示。

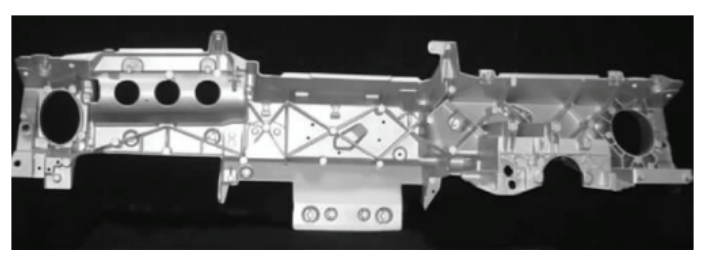

图 3 别克君悦车型的压铸镁仪表板梁

\section{5 轻量化技术在农机中的应用}

汽车轻量化已经成为全球各大车企提高产品竞 争力的一个重要手段, 也是未来汽车工业发展的重 要趋势。目前, 关于汽车轻量化技术的研究已取得 很多成果, 在不同车型上进行了应用。农业机械作 为非道路移动车辆, 在作业时排放的尾气 $(\mathrm{CO}$ 、 NOx、PM 等)严重影响空气质量, 对生态环境构成 威胁, 因此, 对农业机械进行轻量化技术开发, 减 少作业时污染气体的排放, 降低燃油消耗, 提高其 作业性能有着重要的现实意义。

吴伟斌等 ${ }^{[71]}$ 采用拓扑优化和尺寸优化方法对 某一山地轮式果园运输车车架进行了轻量化设计, 首先通过静态应变实验验证了有限元分析结果的准 确合理性, 然后基于满载弯曲工况和满载扭转工况 下的静态力学分析结果和前八阶模态分析结果对车 架的横梁位置和梁厚度进行了优化改进(外横梁由 $5 \mathrm{~mm}$ 减薄至 $4.6034 \mathrm{~mm}$, 其余梁厚度由 $5 \mathrm{~mm}$ 减薄 至 $3.5659 \mathrm{~mm}$ ), 在满足强度、刚度以及模态性能要 求的情况下, 成功实现了 $12.4 \%$ 的减重目标, 优化 
前后车架的性能参数对比结果如表 3 所示。

表 3 车架优化前后各性能参数对比

\begin{tabular}{ccccc}
\hline 车架性能参数 & 优化前 & 优化后 & 变化量 & 变化率 $(\%)$ \\
\hline 质量 $/ \mathrm{kg}$ & 103 & 90.26 & -12.74 & -12.4 \\
弯曲最大等效应力 $/ \mathrm{MPa}$ & 40.19 & 60.77 & 20.58 & 51.2 \\
弯曲最大变形量 $/ \mathrm{mm}$ & 0.371 & 0.483 & 0.112 & 30.2 \\
扭转最大等效应力 $/ \mathrm{MPa}$ & 56.8 & 65.394 & 8.594 & 15.1 \\
扭转最大变形量 $/ \mathrm{mm}$ & 2.985 & 3.28 & 0.295 & 9.9 \\
一阶扭转频率 $/ \mathrm{Hz}$ & 83.234 & 76.478 & -6.756 & -8.1 \\
一阶弯曲频率/ $\mathrm{Hz}$ & 85.571 & 80.501 & -5.07 & -5.9 \\
\hline
\end{tabular}

张娜娜等 ${ }^{[72]}$ 基于试验工况、正常工况和极限 工况三种工况下的应力应变分析结果, 综合采用 尺寸优化 (5 个厚度尺寸参数) 和结构优化方法对 某高速水稻插秧机车架进行轻量化设计, 最后在 保证车架的刚度和强度要求的同时, 实现了 $16.77 \%$ 的减重效果。焦晨龙 ${ }^{[73]}$ 基于 Hyperworks 软件对 JN4LZ履带式联合收割机机体进行轻量化 设计, 利用 Optistruct 模块对机体进行拓扑和尺寸 优化, 最终在满足机体强度和刚度要求的同时, 实现了机体 $119 \mathrm{~kg}$ 的减重。藏世宇等 ${ }^{[74]}$ 基于 ANSYS Workbench 软件对某谷物联合收割机脱 粒机机架进行轻量化设计, 使用拓扑优化方法使 机架质量成功减少了 $20 \%$ 。韩红阳等 ${ }^{[75]}$ 使用尺寸 优化的方法对某机动式喷杆喷雾机架进行轻量化 设计成功实现 $40.4 \%$ 的减重效果, 并且依据轻量 化设计结果对机架进行结构优化设计, 一定程度 地提高了机架的安全系数。冯伟等 ${ }^{[76]}$ 利用 ISIGHT 软件对某小型收割机机架进行了轻量化设计, 首 先试验模态验证了仿真分析模态的合理性, 基于 上述分析结果对机架型材的 7 个壁厚参数进行优 化, 在保证最大应力不变的条件下, 机架成功减 重 $11 \mathrm{~kg}$, 实现了机架的 $22.9 \%$ 的减重目标。周方 思等 ${ }^{[77]}$ 基于 ANSYS Workbench 软件采用拓扑优 化和尺寸优化两级优化方案对某除草机车架进行 轻量化设计, 在保证车架强度以及刚度性能的同 时, 实现了车架的 $33.89 \%$ 的减重效果。袁守利等 ${ }^{[78]}$ 采用尺寸优化方法对 3WPZ-500 自走式喷杆喷雾机 架进行轻量化设计, 以 13 个横纵梁的厚度为设计 变量, 优化后的机架质量 $(28.57 \mathrm{~kg})$ 较优化前 (68.22 kg)减少 $39.65 \mathrm{~kg}$, 减重率高达 $58.1 \%$, 且 满足强度和刚度要求。陶雷等 ${ }^{[79]}$ 综合使用尺寸和 结构优化方法对某秥秆打捆机的机架进行轻量化
设计, 最终在满足机架静强度要求、固有频率要 求和振动要求的同时, 使得机架质量减轻 $19.481 \mathrm{~kg}$, 减重率达 $15.51 \%$ 。舒成松等 ${ }^{[80]}$ 采用拓 扑优化和多目标优化方法对某烟秆拔秆破碎机机 架进行轻量化设计, 在满足机架强度、刚度要求 的同时, 实现减重 $18.78 \mathrm{~kg}$, 减重率达 $13.09 \%$ 。 周林 ${ }^{[81}$ 基于 ANSYS Workbench 软件对某玉米收 获机底盘车架进行强度分析, 并且对车架的疲劳 寿命进行了分析, 依据疲劳寿命分析结果采用形 状优化方法对车架的关键部件进行了轻量化设 计, 最终实现了对该部件的 $24 \%$ 减重目标。鄢强 等 ${ }^{[82]}$ 利用拓扑优化方法对某一谷物脱粒机机架 进行轻量化设计, 得到了在满足静力学强度和刚 度的要求下机架减重 $10.98 \%$ 的结果。李耀明等 ${ }^{[83]}$ 在对沃得锐龙 4LZ-2.5B 型履带式联合收获机割 台进行参数优化设计时, 首先通过模态试验验证 了有限元模态分析结果的合理性, 同时发现割台 机架与脱粒滚筒发存在共振激励现象, 针对上述 现象利用尺寸优化方法对割台的横梁、弯梁、底 板、侧板的厚度进行改进, 通过正交试验设计确 定了四个参数的最佳组合(横、弯梁由 $3 \mathrm{~mm}$ 减薄 至 $2.8 \mathrm{~mm}$, 底板、侧板由 $1.2 \mathrm{~mm}$ 减薄至 $0.8 \mathrm{~mm}$ ), 在满足强度和刚度要求同时, 有效地解决了机架 与滚筒的共振现象, 实现割台减重 $17.56 \mathrm{~kg}$, 减 重率达 $14.02 \%$ 。生所等 ${ }^{[84]}$ 在对双轴旋耕机机架进 行轻量化设计时, 采用结构优化方法分别对侧板 和挡泥板进行再设计, 实现了对侧板、挡泥板的 $44.8 \% 、 37 \%$ 的减重效果。谢斌等 ${ }^{[85}$ 基于实测载荷 采用多目标拓扑优化方法对某蔬菜田间作业机械 车架进行了轻量化设计, 在保证车架可靠性的前 提下, 实现车架减重 $443.55 \mathrm{~kg}$, 减重率高达 $53.47 \%$, 优化前后车架结构如图 4 所示。

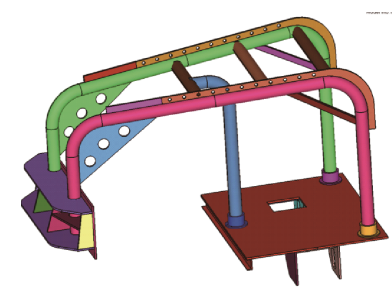

(a) 优化前

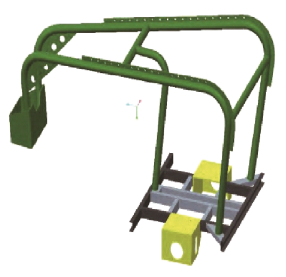

(b) 优化后
图 4 车架结构示意图

王利鹤等 ${ }^{[86]}$ 以减轻重量和提高刚度为目标对 对某深松机机架进行了拓扑优化设计, 优化后的机 架质量减轻 $39.03 \%$, 对优化后的机架进行了静力学 和模态分析, 发现机架的最大应力 $177.79 \mathrm{MPa}$, 较 优化前(258.81 MPa)降低 $31.3 \%$, 且模态性能满足设 
计要求。刘柄昌 ${ }^{[87]}$ 使用拓扑优化对某设施蔬菜秸秆 还田装备支架进行轻量化设计, 结果显示, 优化后 的支架不仅在强度上和优化前相比无明显变化, 而 且实现了 $22.6 \%$ 的减重目标。黄琼春等 ${ }^{[88]}$ 采用尺寸 优化方法对某小型甘蔗机台架结构进行轻量化设 计, 最终在实现台架 $12 \mathrm{~kg}$ 减重的同时, 提高了台 架的刚性。龙超等 ${ }^{[89}$ 基于尺寸优化对某菱形收割机 车架进行轻量化设计, 在保证车架强度的同时, 实 现了 $58 \mathrm{~kg}$ 的减重目标。孙潇鹏等 ${ }^{[90]}$ 利用结构优化 和材料替换方法对某水稻收割机的割台进行轻量化 设计, 经轻量化设计后的割台比原割台质量减少 $49.872 \mathrm{~kg}$, 并一定程度地提高了收割机的燃油经济 性。徐敏雅等 ${ }^{[91]}$ 基于 ANSYS 软件对某拖拉机的下 拉杆进行拓扑优化设计, 结果显示, 优化后的下拉 杆质量较优化前降低 $29.2 \%$ 。范思宇等 ${ }^{[92]}$ 基于结构 优化设计对某果园电动履带运输车车架进行轻量化 设计, 结果表明, 在满足强度和刚度的基础上, 优 化设计后的车架质量减轻 $8.82 \%$, 且模态性能得到 了提高。胡建平等 ${ }^{[93]}$ 基于 ANSYS 软件对某移栽机 的提升支架进行轻量化设计, 基于敏感度分析对篮 选出的敏感参数进行优化设计, 结果表明, 优化设 计后的支架质量减轻 $37.38 \%$, 且强度得到了很大的 提升。廖宇兰等 ${ }^{[94]}$ 基于灵敏度分析使用尺寸优化方 法 (以前梁 $x_{1}$ 、中间梁 $x_{2}$ 、加强梁 $x_{3}$ 、后梁 $x_{4}$ 和底 梁 $x_{5}$ 厚度为设计变量, 初始值均为 $10 \mathrm{~mm}$ )对某木薯 收获机机架进行优化设计, 通过模糊物元法获得了 最优设计方案 $\left(x_{1}=10.74 \mathrm{~mm}, x_{2}=12.07 \mathrm{~mm}\right.$, $x_{3}=12.8 \mathrm{~mm}, x_{4}=7.29 \mathrm{~mm}, x_{5}=6.94 \mathrm{~mm}$ ), 结果表明, 最佳优化设计方案较原始方案质量减轻 $4.29 \%$, 且 最大变形减少 $27.04 \%$, 静态刚度性能得到了很好的 改善。沈伟等 ${ }^{[95}$ 综合使用变密度法拓扑优化和尺寸 优化方法(通过响应面法确定了五个尺寸优化设计 变量的最佳设计方案)对某收割机变速箱壳体进行 轻量化设计, 在满足壳体强度和刚度的条件下, 使 得壳体质量减少 $1.78 \mathrm{~kg}$, 减重率达 $17.1 \%$ 。储婷婷 等 ${ }^{[96]}$ 基于尺寸优化方法对某分体式插种机种箱进 行轻量化设计研究, 实现了对秧箱的 $1.4 \mathrm{~kg}$ 减重, 减重率达 $9.59 \%$ 。胡奔等 ${ }^{[97]}$ 使用拓扑优化和尺寸优 化方法对某拖拉机变速箱壳体进行轻量化设计, 结 果显示, 在满足强度和刚度要求下, 两级优化后的 壳体质量较优化前减轻 $16.3 \%$ 。赵知辛等 ${ }^{[98]}$ 在对某 小麦脱皮机转子进行轻量化设计时, 考虑对转子主 轴内直径和转子横梁截面尺寸(图 5)进行优化设计, 通过响应面法(RSM)得到了优化变量最佳参数组合 (表 4), 实现了对脱皮机转子的 $73.6 \mathrm{~kg}$ 减重, 减重
率达 $35.16 \%$ 。

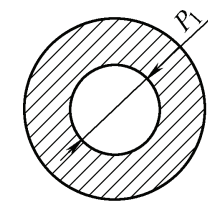

(a) 主轴

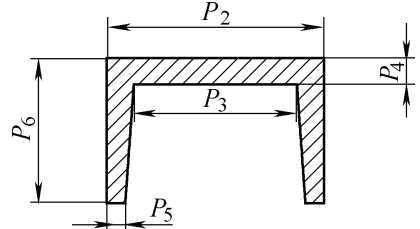

(b) 转子横梁截面
图 5 尺寸优化变量示意图

表 4 优化前后参数对比

\begin{tabular}{cccc}
\hline 优化变量 & 优化前 & 优化后 & 变化量(\%) \\
\hline$P_{1} / \mathrm{mm}$ & 27.5 & 31.027 & 12.8 \\
$P_{2} / \mathrm{mm}$ & 27.5 & 25.191 & -8.4 \\
$P_{3} / \mathrm{mm}$ & 27.5 & 25.613 & -6.9 \\
$P_{4} / \mathrm{mm}$ & 27.5 & 6.037 & -78.0 \\
$P_{5} / \mathrm{mm}$ & 27.5 & 4.667 & -83.0 \\
$P_{6} / \mathrm{mm}$ & 27.5 & 46.292 & 68.3 \\
\hline
\end{tabular}

毛鹏军等 ${ }^{[99]}$ 基于 ANSYS Workbench 软件使用 尺寸优化方法对某烟田辅助采收机车架进行轻量化 优化设计, 优化结果显示, 车架质量减轻 $4.127 \mathrm{~kg}$, 减重率达 $18.77 \%$, 并且通过有限元仿真分析验证了 优化后的车架的可靠性。潘群林 ${ }^{[100]}$ 在对某丘陵山地 拖拉机前转向驱动桥进行轻量化研究时, 使用尺寸 优化方法对前驱动桥壳和前转向节进行了优化设 计, 实现分别减重 $4.2 \mathrm{~kg} 、 1.5 \mathrm{~kg}$, 总共减重 $5.7 \mathrm{~kg}$, 减重率达 5\%。吴锦标 ${ }^{[101]}$ 使用尺寸优化方法对某喷 雾机的内、外喷杆进行轻量化设计, 分别实现了对 内、外喷杆减重 $72.46 \mathrm{~kg} 、 52.9 \mathrm{~kg}$, 减重率分别为 $59.5 \% 、 13.69 \%$, 减重效果显著。张洪伟等 ${ }^{[102]}$ 基于 有限元分析软件 ANSYS 使用尺寸优化方法 8 个管 厚度尺寸为设计变量)对某农用车车架进行了优化 设计, 最终使得车架的质量减小 $20 \%$ 的同时提高了 车架的模态性能, 且优化后的后悬架刚度由 $62500 \mathrm{~N} / \mathrm{m}$ 提高至 $60800 \mathrm{~N} / \mathrm{m}$ 。YANG 等 ${ }^{[103]}$ 使用拓 扑优化方法对某并联混合动力拖拉机的后驱动桥进 行了轻量化设计, 优化结果显示, 新方案较原方案 质量减轻 $22.7 \%$, 且强度和刚度都得到了较大的提 高。郑斌等 ${ }^{[104]}$ 使用尺寸优化方法对一高压共轨柴油 机的连杆进行了优化设计, 结果显示, 优化后的连 杆体积较优化前减少 $3.4 \%$, 且满足强度和刚度要 求。陈远帆等人 ${ }^{[105]}$ 综合使用拓扑优化和尺寸优化方 法对某割草车车架进行轻量化设计, 结果表明, 经 两级优化后的车架质量减轻 $23.022 \mathrm{~kg}$, 减重率达 $34.3 \%$, 取得了较显著的轻量化效果。赵韩等 ${ }^{[106]}$ 利 用尺寸优化方法对某半挂车车架进行了轻量化设 
计, 在满足车架强度和刚度要求的同时, 实现对车 架减重 $1.13 \mathrm{t}$, 减重率达 $18.4 \%$ 。马丽娜等 ${ }^{[107]}$ 在对 某轮式联合收获机桥壳进行轻量化优化设计时, 对 桥壳的截面形状和尺寸进行了优化设计, 使得桥壳 的质量减轻 $17.8 \%$, 并且通过仿真分析验证了优化 后的桥壳满足强度和刚度要求。邓若玲等 ${ }^{[108]}$ 基于折 衷规划多目标拓扑优化方法对某拖拉机变速箱箱体 进行轻量化设计研究, 在实现箱体减重 $147.8 \mathrm{~kg}$ (减 重率达 $41 \%$ )的同时, 提高了箱体的强度和刚度(最 大应力由 $441 \mathrm{MPa}$ 减至 $298 \mathrm{MPa}$, 最大形变量由 $0.219 \mathrm{~mm}$ 减至 $0.158 \mathrm{~mm}$ ), 优化结果如图 6 所示。

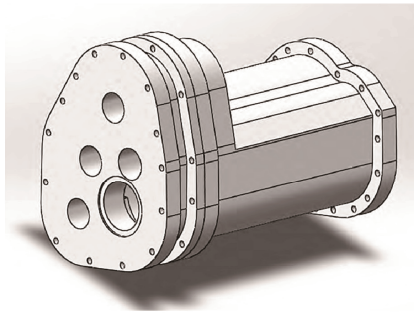

(a) 优化前

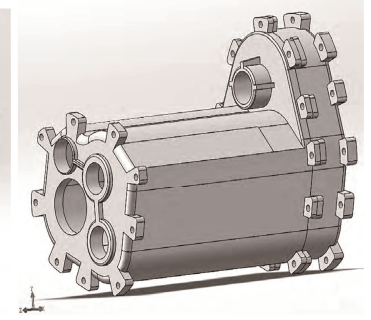

(b) 优化后
图 6 箱体结构示意图

张毛宁等 ${ }^{[109]}$ 利用拓扑优化方法对某辊式磨粉 机机架进行了优化设计, 在不降低机架的强度和刚 度性能的基础上，实现了对机架的 12\%减重效果。 李怡等 ${ }^{[110]}$ 利用尺寸优化方法(以底盘、驾驶室纵梁、 发动机室、机架纵梁、机架横梁为尺寸优化设计变 量)对某大豆收获机车架进行了轻量化设计, 底盘减 薄 $1 \mathrm{~mm}$, 驾驶室纵梁增厚 $0.2 \mathrm{~mm}$, 发动机室减薄 $1 \mathrm{~mm}$, 机架纵梁减薄 $0.3 \mathrm{~mm}$, 机架横梁减薄 $1 \mathrm{~mm}$, 优化后的车架在满足强度要求的同时, 较优化前减 轻 $29.7 \mathrm{~kg}$, 减重率达 $16 \%$, 该轻量化设计方案得到 了企业的采纳实施。李海亮等 ${ }^{[111]}$ 利用尺寸优化方法 对某播种机折叠式机架进行了优化设计, 优化后的 机架较优化前减轻 $101.53 \mathrm{~kg}$, 减重率达 $13.5 \%$, 且 满载下的最大变形量和最大应力值都有所下降。周 成军等 ${ }^{[12]}$ 设计了一穿轮配重轻型跑车并且对跑车 的两块挡板进行了尺寸优化设计, 使得跑车质量减 轻 $9.3 \mathrm{~kg}$, 减重率达 $18.6 \%$ 。赵映 ${ }^{[113]}$ 以某栽种车车 架为研究对象, 基于有限元分析结果对车架的梁截 面尺寸和结构进行了两次优化改进, 结果表明, 经 两次优化后的车架较原车架质量减轻 $12.9 \mathrm{~kg}$, 减重 率达 $22.7 \%$, 且车架的力学性能得到了良好的改善。 许学建 ${ }^{[14]}$ 设计了一履带式高地隙茶园管理机行驶 底盘系统, 通过有限元分析发现底盘系统的最大应 力远小于材料的屈服应力(强度富余), 针对上述问 题, 使用尺寸优化方法对底盘系进行了两次优化设
计, 实现底盘系减重 $44 \mathrm{~kg}$, 减重率达 $8.3 \%$ 。雷哓 晖等 ${ }^{[115]}$ 对某背负式割草机的常使用的 3 种刀盘进 行了拓扑优化设计，3 种刀盘经优化后质量分别减 轻 $27.5 \% 、 10.24 \% 、 12.71 \%$, 并且通过试验验证了 刀盘的各项性能指标均满足行业标准。李再参等 ${ }^{[16]}$ 对某旋耕机机架进行了轻量化设计, 使用尺寸优化 方法对机架的横梁的厚度进行了优化设计, 在满足 机架强度和刚度要求的前提下, 实现了机架最大减 重 $34 \mathrm{~kg}$, 减重率达 $30 \%$ 。张旻轩 ${ }^{[117]}$ 使用尺寸优化 方法对某农林拖拉机的两柱式 ROPS 进行了优化设 计, 使得新结构较原结构减重 $23.75 \%$, 且通过仿真 分析和纵向加载试验、压垮试验、侧向加载试验验 证了轻量化设计方案的合理性。DU 等 ${ }^{[118]}$ 在对某收 获机轴流滚筒进行轻量化设计时, 基于静力学和模 态仿真分析结果, 对通过灵敏度分析获得的设计变 量进行优化设计 (3 个尺寸参数变量), 实现了滚筒减 重 $24 \mathrm{~kg}$, 减重率达 $15 \%$, 同时提高了滚筒的整体 性能。

综上, 国内外关于农业机械轻量化研究主要集 中在结构优化设计方面。基于现代发展较为成熟的 有限元仿真技术, 利用结构优化手段对农业机械进 行轻量化设计, 具有可操作性强且节约成本的优 点 ${ }^{[119]}$ 。因此, 使用尺寸优化、拓扑优化等结构优化 手段对农业机械进行轻量化设计得到了广泛的应 用。随着农业装备的持续发展, 未来轻量化材料和 轻量化工艺技术在农业机械轻量化领域的应用将逐 步得到深入研究 ${ }^{[120]}$ 。借鉴汽车轻量化领域现已取得 的研究成果, 结合农业装备实际作业环境和要求, 探索从结构、材料和工艺方面对农业机械进行轻量 化技术开发是未来的发展趋势。

\section{6 结论与展望}

轻量化是一个集成的复杂系统工程, 结合计算 机辅助工程(CAE)采用各种先进的优化算法(基因遗 传算法、改进粒子群算法等)从结构设计出发可以得 到较好的轻量化效果; 使用轻质材料是实现轻量化 的最有效、最直接的途径; 针对异种材料连接以及 材料成形问题, 采用先进的连接技术和成形工艺可 以进一步提升轻量化效果, 要想取得最佳的轻量化 系数, 必须同时考虑新结构、新材料和新工艺三个 方面的内容。农业机械轻量化技术展望如下。

（1）结构优化设计方面：尺寸优化方法的应用 已经比较成熟, 形状优化存在一定的局限性, 拓扑 优化和多学科、多目标设计优化是未来重点研究发 
展方向。

(2) 轻量化材料方面: 多材料混合车身是未来 发展的必然趋势, 也是实现轻量化的理想途径, 高 强度钢、铝合金以及塑料和复合材料等轻质材料的 使用将日益增加。

(3) 制造工艺方面: 随着用材日益混合多样化, 对制造工艺要求也越来越高, 未来激光焊接技术、 液压成形、热冲压成形、铸造成形都将在轻量化中 发挥重要作用。

(4) 轻量化技术在农机中的应用将日益增强, 随着世界各国排放标准的提高以及我国非道路移动 机械第四阶段排放标准的出台，未来各种农机制造 必然要朝着轻量化方向发展。

\section{参 考 文 献}

[1] 李永兵, 马运五, 楼铭, 等. 轻量化多材料汽车车身连 接技术进展 $[\mathrm{J}]$. 机械工程学报，2016，52(24)：1-23.

LI Yongbing, MA Yunwu, LOU Ming, et al. Advances in welding and joining processes of multi-material lightweight car body[J]. Journal of Mechanical Engineering, 2016, 52(24): 1-23.

[2] 范子杰, 桂良进, 苏瑞意. 汽车轻量化技术的研究与进 展 $[\mathrm{J}]$. 汽车安全与节能学报, 2014，5(1)：1-16.

FAN Zijie, GUI Liangjin, SU Ruiyi. Research and development of automotive lightweight technology[J]. Journal of Automotive Safety and Energy, 2014, 5(1): $1-16$.

[3] 李永兵, 李亚庭, 楼铭, 等. 轿车车身轻量化及其对连 接技术的挑战[J]. 机械工程学报, 2012, 48(18): 44-54. LI Yongbing, LI Yating, LOU Ming, et al. Lightweighting of car body and its challenges to joining technologies[J]. Journal of Mechanical Engineering, 2012, 48(18): 44-54.

[4] 李光雯, 刘新玲. 汽车轻量化技术的研究现状综述 $[\mathrm{J}]$. 材料科学与工艺，2020，28(5)：47-61.

LI Guangji, LIU Xinling. Literature review on research and development of automotive lightweight technology[J]. Materials Science and Technology, 2020, 28(5): 47-61.

[5] 赵晓东, 穆希辉, 杜峰坡, 等. 汽车结构轻量化的研究 与进展 $[\mathrm{J}]$. 现代制造工程, 2017(10): 157-162.

ZHAO Xiaodong, MU Xihui, DU Fengpo, et al. Research and development of automotive structure lightweight[J]. Modern Manufacturing Engineering, 2017(10): 157-162.

[6] 左文杰, 陈继顺, 李亦文, 等. 刚度、强度与频率约束 下的白车身板厚尺寸优化 [J]. 汽车工程, 2017，39(2): 145-149.
ZUO Wenjie, CHEN Jishun, LI Yiwen, et al. Size optimization on plate thickness of BIW with constraints of stiffness , strength and frequency[J]. Automotive Engineering, 2017, 39(2): 145-149.

[7] 于志新, 曹全德, 杨士通. 某载货汽车车架有限元分析 及尺寸优化[J]. 机械工程师, 2018(1): 18-20.

YU Zhixin, CAO Quande, YANG Shitong. Finite element analysis and dimension optimization of a truck frame[J]. Mechanical Engineer, 2018(1): 18-20.

[8] 宋晓飞, 林荣会, 李帅朝, 等. 基于 OptiStruct 的驱动 桥壳轻量化设计 $[\mathrm{J}]$. 机械传动, 2019，43(4): 83-88, 97.

SONG Xiaofei, LIN Ronghui, LI Shuaichao, et al. Lightweight design of drive axle housing based on OptiStruct[J]. Journal of Mechanical Transmission, 2019, 43(4): 83-88, 97.

[9] 黄纬, 廖美渘, 郭绍良, 等. 某汽车后悬架下摆臂尺寸 优化设计 [J]. 客车技术与研究，2019，41(2)：28-30， 51.

HUANG Wei, LIAO Meiying, GUO Shaoliang, et al. Optimization design of lower swing arm sizes for a vehicle rear suspension[J]. Bus \& Coach Technology and Research, 2019, 41(02): 28-30, 51.

[10] 王震虎, 王万林, 张松波, 等. 基于车身概念模型的白 车身主断面尺寸优化 [J]. 汽车工程, 2018，40(8): 904-911.

WANG Zhenhu, WANG Wanling, ZHANG Songbo, et al. Size optimization on main cross-sections of body-in-white based on conceptual model for car body[J]. Automotive Engineering, 2018, 40(8): 904-911.

[11] 周琪, 马其华, 周天俊. 承载式碳纤维缠绕内祄钢管车 架轻量化设计 $[J]$. 现代制造工程, 2020(4): 57-63.

ZHOU Qi, MA Qihua, ZHOU Tianjun. Lightweight design of load-bearing frame with CFRP winding steel tube[J]. Modern Manufacturing Engineering, 2020(4): 57-63.

[12] LI S Q, FENG X Y. Study of structural optimization design on a certain vehicle body-in-white based on static performance and modal analysis[J]. Mechanical Systems and Signal Processing, 2020, 135: 106405-106415.

[13] HOU W B, XU X, HAN X Z, et al. Multi-objective and multi-constraint design optimization for hat-shaped composite T-joints in automobiles[J]. Thin-Walled Structures, 2019, 143: 106232-106242.

[14] 姜丽红, 吴庆捷. 某前副车架性能分析与轻量化设计 $[\mathrm{J}]$. 
机械强度，2020，42(6): 1503-1508.

JIANG Lihong, WU Qingjie. Performance analysis and lightweight design of a front subframe[J]. Journal of Mechanical Strength, 2020, 42(6): 1503-1508.

[15] 龚旭, 谷正气, 李振磊, 等. 基于近似模型的集装箱半 挂车导流罩的形状优化 [J]. 汽车工程, 2011, 33(1): 38-42.

GONG Xu, GU Zhengqi, LI Zhenlei, et al. Shape optimization of wind deflector in container semitrailer based on approximate model[J]. Automotive Engineering, 2011, 33(1): 38-42.

[16] 廖萝, 李峰, 李志. 概念设计阶段铝合金后副车架轻量 化设计 $[\mathrm{J}]$. 汽车工程, 2020，42(12): 1737-1743.

LIAO Ying, LI Feng, LI Zhi. Lightweight design of aluminum rear subframe in conceptual design stage[J]. Automotive Engineering, 2020，42(12): 1737-1743.

[17] 陈静, 彭博, 王登峰, 等. 碳纤维增强复合材料电池箱 轻量化设计 [J]. 汽车工程, 2020, 42(2): 257-263, 277. CHEN Jing, PENG Bo, WANG Dengfeng, et al. Lightweight design of Carbon Fiber Reinforced Composite battery box[J]. Automotive Engineering, 2020, 42(2): 257-263, 277.

[18] 陆善涁, 蒋伟波, 左文杰. 基于等效静态载荷法的汽车 前端结构抗撞性尺寸和形貌优化 [J]. 振动与冲击, 2018, 37(7): 56-61.

LU Shanbin, JIANG Weibo, ZUO Wenjie. Size and morphology crash-worthiness optimization for automotive frontal structures using equivalent static loads method[J]. Journal of Vibration and Shock, 2018，37(7): 56-61.

[19] 张楠, 孙章栋, 任爱华, 等. 基于拓扑优化的电动汽车 变速器箱体轻量化设计 $[\mathrm{J}]$. 湖北汽车工业学院学报, 2020, 34(4): 16-19.

ZHANG Nan, SUN Zhangdong, REN Aihua, et al. Lightweight design of electric vehicle gearbox body based on topology optimization[J]. Journal of Hubei University of Automotive Technology, 2020，34(4): 16-19.

[20] 刘洋, 申加伟, 王超, 等. 基于 Inspire 软件的复合支 架轻量化拓扑优化设计 $[\mathrm{J}]$. 内燃机与动力装置, 2020 , 37(6): 7-12.

LIU Yang, SHEN Jiawei, WANG Chao, et al. Lightweight design on composite bracket based on topology optimization by Inspire[J]. Internal Combustion Engine \& Powerplant, 2020, 37(6): 7-12.

[21] 李雨宣. 某重型汽车平衡悬架轻量化设计[D]. 重庆: 重 庆理工大学, 2019 .
LI Yuxuan. Lightweight design of balance suspension for a heavy vehicle[D]. Chongqing: Chongqing University of Technology, 2019.

[22] 周思, 吴小冬, 潘嵩. 汽车前转向节铝合金轻量化拓扑 优化设计 $[J]$. 汽车实用技术, 2019(19): 149-151， 160 . ZHOU Si, WU Xiaodong, PAN Song. Lightweight topology optimization design of aluminum alloy for front steering knuckle[J]. Automobile Applied Technology, 2019(19): 149-151, 160.

[23] VIQARUDDIN M, REDDY D. Structural optimization of control arm for weight reduction and improved performance[J]. Materials Today: Proceedings, 2017, 4(8): 9230-9236.

[24] JUNG Y, LIM S, KIM J, et al. Lightweight design of electric bus roof structure using multi-material topology optimization[J]. Structural and Multidisciplinary Optimization, 2020, 61(3): 1 .

[25] 王战, 问瑞雷, 黄向东. 基于多学科设计优化的悬架互 换研究 $[J]$. 汽车工程, 2016, 38(6): 738-744.

WANG Yu, YAN Ruilei, HUANG Xiangjun. A research on suspension exchange based on multidisciplinary design optimization[J]. Automotive Engineering, 2016，38(6): 738-744.

[26] 史朝军, 张胜兰, 杨啓梁. 自卸车货箱多学科优化设 计 $[\mathrm{J}]$. 湖北汽车工业学院学报, 2013, 27(3): 10-14. SHI Chaojun, ZHANG Shenglan, YANG Qiliang. Multidisciplinary optimization for cargo box of dumper[J]. Journal of Hubei University of Automotive Technology, 2013, 27(3): 10-14.

[27] 史国宏, 陈勇, 杨雨泽, 等. 白车身多学科轻量化优化 设计应用[J]. 机械工程学报, 2012, 48(8): 110-114. SHI Guohong, CHEN Yong, YANG Yuze, et al. BIW architecture multidisciplinary light weight optimization design[J]. Journal of Mechanical Engineering, 2012, 48(8): $110-114$.

[28] 胡朝辉, 成艾国, 钟志华. 多学科优化设计在热成形车 架轻量化中的应用 $[\mathrm{J}]$. 中国机械工程, 2010, 21(6): 728-732.

HU Chaohui, CHEN Aiguo, ZHONG Zhihua. Research on application of multidisciplinary design optimization for hot stamp body frame lightweight[J]. China Mechanical Engineering, 2010, 21(6): 728-732.

[29] 刘钊, 李晗, 朱平. 基于粒子群算法的轿车车身多学科 优化设计 [J]. 汽车工程, 2018, 40(3): 251-258.

LIU Zhao, LI Han, ZHU Ping. Multidisciplinary design 
optimization of car body based on particle swarm algorithm[J]. Automotive Engineering, 2018, 40(3): 251-258.

[30] 于成龙, 刘䒯, 乔金金. 基于多学科优化的锂离子动力电 池包轻量化设计 $[\mathrm{J}]$. 汽车安全与节能学报, 2019, 10(2): 233-240.

YU Chenglong, LIU Ying, QIAO Xin. Lightweight design of lithium-ion power battery pack based on multidisciplinary optimization[J]. Journal of Automotive Safety and Energy, 2019， 10(2): 233-240.

[31] 高大威, 邓有志, 高云凯. 超高强度钢车门防撞杆结构 多学科优化 [J]. 公路交通科技, 2012, 29(6): 144-149, 158.

GAO Dawei, DENG Youzhi, GAO Yunkai. Multidisciplinary optimal design for Ultra-high-strength steel side impact bar[J]. Journal of Highway and Transportation Research and Development, 2012, 29(6): 144-149, 158.

[32] XIONG F, WANG D, MA Z , et al. Structure-material integrated multi-objective lightweight design of the front end structure of automobile body[J]. Springer Berlin Heidelberg, 2018, 57(2): 829-847.

[33] MIAO B R, LUO Y X., PENG Q M, et al. Multidisciplinary design optimization of lightweight carbody for fatigue assessment[J]. Materials \& Design, 2020, 194: 108910-108921.

[34] 李晓林, 肖宝亮, 缪成亮, 等. 卡车驱动桥壳用轻质高 强度钢的研发 $[\mathrm{J}]$. 上海金属, 2020，42(5): 9-14. LI Xiaolin, XIAO Baoliang, MIAO Chengliang, et al. Development of lightweight high-strength steels for truck drive axle housing[J]. Shanghai Metals, 2020, 42(5): 9-14.

[35] 高新华, 陈云霞. 某轿车门槛梁轻量化设计及回弹控 制[J]. 合肥工业大学学报, 2014，37(2): 150-154.

GAO Xinhua, CHEN Yunxia. Lightweight design for threshold beam of a car and the control of springback[J]. Journal of Hefei University of Technology, 2014，37(2): $150-154$

[36] 周森. 高强度调质钢液化石油气运输半挂车罐体轻量 化设计[D]. 广州: 华南理工大学, 2018 .

ZHOU Sen. Lightweight design of LPG Semi-trailer's tank using high strength quenched and tempered steel[D]. Guangzhou: South China University of Technology, 2018.

[37] 洪腾蛟, 董福龙, 丁凤娟, 等. 铝合金在汽车轻量化领 域的应用研究 $[J]$. 热加工工艺, 2020, 49(4): 1-6. HONG Tengjiao, DONG Fulong, DING Fengjuan, et al.
Application of aluminum alloy in automotive lightweight[J]. Hot Working Technology, 2020， 49(4): $1-6$.

[38] 汽车轻量化技术创新战略联盟. 汽车轻量化用铝合金 底盘件技术开发及产业化应用[EB/OL]. [2020-05-12]. http: //www.calasae.org.cn/xiangmuxiangqing.html.

China Auto Lightweight Technology Innovation Strategic Alliance.Technology development and industrial application of aluminum alloy chassis parts for automobile lightweight[EB/OL]. [2020-05-12].http : // www.calasae.org.cn/xiangmuxiangqing.html.

[39] 林佳武, 李玄霜, 陈宗明, 等. 真空高压铸造铝合金车 身后纵梁轻量化设计 $[\mathrm{J}]$. 汽车工程, $2020,42(3)$ : 383-389, 400.

LIN Guiwu, LI Xuanshuang, CHEN Zongming, et al. Lightweight design of body rear longitudinal beam of VAHP Die-casting aluminum alloy[J]. Automotive Engineering, 2020, 42(3): 383-389, 400.

[40] 王俊峰, 魏震, 陈美玉. 基于性能要求的发动机舱盖轻 量化设计 $[\mathrm{J}]$. 汽车工程师，2020(3): 29-31， 48.

WANG Junfeng, WEI Zhen, CHEN Meiyu. Lightweight design of bonnet based on performance requirements $[\mathrm{J}]$. Auto Engineer, 2020(3): 29-31, 48.

[41] 金泉军, 陈伟, 张然然, 等. 汽车铝质防撞梁的轻量化 设计及分析 $[\mathrm{J}]$. 汽车实用技术，2018(7)：151-156.

JIN Quanjun, CHEN Wei, ZHANG Ranran, et al. Lightweight design and analysis of automobile aluminum alloy anti-collision beam[J]. Automobile Applied Technology, 2018(7): 151-156.

[42] 刘雅芳, 董万鹏, 饶轮. 基于 LS-DYNA 的 6082 铝合 金车门防撞杆轻量化设计与碰撞性能分析 $[\mathrm{J}]$. 上海工 程技术大学学报，2015，29(4): 312-315.

LIU Yafang, DONG Wanpeng, RAO Lun. Lightweight design and crash performance analysis of 6082 aluminum alloy side door beam based on LS-DYNA[J]. Journal of Shanghai University of Engineering Science, 2015, 29(4): 312-315.

[43] 张琼, 李晓光, 鲁飞, 等. 纯电动客车铝合金车身骨架 多目标轻量化设计 $[\mathrm{J}]$. 黄河科技学院学报, 2020, 22(2): 33-39.

ZHANG Qiong, LI Xiaoguang, LU Fei, et al. Multiobjective lightweight design of aluminum alloy body frame for pure electric bus[J]. Journal of Huanghe S\&T College, 2020, 22(2): 33-39.

[44] 赵金金. 镁合金在车辆结构构件上的应用 $[\mathrm{J}]$. 科技与企 
业, 2016(7): 210-211.

ZHAO Xin. Application of magnesium alloy in vehicle structural components $[\mathrm{J}]$. Technology and Enterprise, 2016(7): 210-211.

[45] 秦超, 姚晨再, 朱雪林. 轻量化材料在汽车上的精准应 用 $[\mathrm{J}]$. 汽车零部件, 2019(6): 100-107.

QIN Chao, YAO Chenran, ZHU Xuelin. Precise application of lightweight materials in automobiles[J]. Automobile Parts, 2019(6): 100-107.

[46] 陈甜斌. 一种镁合金座椅骨架的强度性能研究 [J]. 汽 车工艺与材料, 2020(9): 20-23.

CHEN Tianbin. Strength property research on a magnesium alloy seat frame[J]. Automobile Technology \& Material, 2020(9): 20-23.

[47] 刘杰, 赵松林. 锻压工艺参数对镁合金汽车车轮耐蚀性 能的影响[J]. 热加工工艺, 2020，49(17): 105-107.

LIU Jie, ZHAO Songlin. Effect of forging process parameters on corrosion resistance of magnesium alloy automotive wheels[J]. Hot Working Technology, 2020, 49(17): 105-107.

[48] LÜ R, JIANG X, MINORU, et al. Lightweight design of automobile frame based on magnesium alloy[J]. IOP Conference Series: Materials Science and Engineering, 2018, 372(1): 1 .

[49] 高景峰, 刘超. 汽车零件用差压铸造 Mg-9Al-1Zn-0.5Ca 镁合金的组织及性能[J]. 热加工工艺, 2020，49(11): 64-66.

GAO Jinfeng, LIU Chao. Microstructure and properties of Mg-9Al-1Zn-0.5Ca magnesium alloy with differential pressure casting for automobile parts[J]. Hot Working Technology, 2020, 49(11): 64-66.

[50] 张妍, 庞有俊, 李杨. 钛合金与汽车轻量化技术 $[\mathrm{J}]$. 时 代汽车, 2019(19): 12-14.

ZHANG Yan, PANG Youjun, LI Yang. Titanium alloy and automobile lightweight technology[J]. Auto Time , 2019(19): 12-14.

[51] 宋鸿玉. 日本新日铁住金公司钛合金应用于雅马哈摩 托车发动机 $[\mathrm{J}]$. 中国钛业, 2015(1): 28.

SONG Fengyu. NSSMC supplied titanium to Yamaha motorcycle engines[J]. China Titanium Industry, 2015(1): 28.

[52] 姚文静. 大众首款 3D 打印钛合金制动钳通过测试 $[\mathrm{J}]$. 中国钛业，2019(1): 49.

YAO Wenjing. Volkswagen tests the world's first 3D printed titanium brake caliper[J]. China Titanium
Industry, 2019(1): 49.

[53] 马捷, 乔冰. 化工新材料在汽车轻量化领域的应用 $[\mathrm{J}]$. 化学工业, 2019, 37(1): 21-29+49.

MA Jie, QIAO Bing. Development of new chemical materials used for automotive light weighting in China[J]. Chemical Industry, 2019, 37(1): 21-29+49.

[54] 王利刚, 李军. 我国汽车轻量化材料“十三五”时期发展 回顾及未来展望[J]. 新材料产业, 2020(6)：25-30.

WANG Ligang, LI Jun. Review of the development of my country's automotive lightweight materials during the 13th Five-Year Plan period and future prospects[J]. Advanced Materials Industry, 2020(6): 25-30.

[55] 陈静, 唐傲天, 田凯, 等. 碳纤维复合材料防撞梁轻量 化设计 [J]. 汽车工程, 2020，42(3): 390-395.

CHEN Jin, TANG Aotian, TIAN Kai, et al. Lightweight design of carbon fiber composite anti-collision beam[J]. Automotive Engineering, 2020，42(3): 390-395.

[56] 王庆, 卢家海, 刘钊, 等. 碳纤维增强复合材料汽车保 险杜的轻量化设计 $[\mathrm{J}]$. 上海交通大学学报, 2017, 51(2): 136-141.

WANG Qing, LU Jiahai, LIU Zhao, et al. A lightweight design of carbon fiber reinforced plastic auto bumper[J]. Journal of Shanghai Jiaotong University, 2017, 51(2): 136-141.

[57] LIU Z, LU J H, ZHU P. Lightweight design of automotive composite bumper system using modified particle swarm optimizer[J]. Composite Structures, 2016, 140: 630-643.

[58] TAO W, LIU Z, ZHU P, et al. Multi-scale design of three dimensional woven composite automobile fender using modified particle swarm optimization algorithm[J]. Composite Structures, 2017, 181: 73-83.

[59] 汪健坤, 李强, 黄磊, 等. 激光焊接技术最新研究进展 及应用现状 $[J]$. 金属加工(热加工), 2020(3)：4-10.

WANG Jiankun, LI Qiang, HUANG Lei, et al. The latest research progress and application status of laser welding technology[J]. MW Metal Forming, 2020(3): 4-10.

[60] 黄林, 陈馨. 激光拼焊板在天窗加强框上的应用 [J]. 锻 压技术，2018，43(6)：28-31.

HUANG Lin , CHEN Xin. Application of laser tailor-welded blanks for sunroof strengthening frame[J]. Forging \& Stamping Technology, 2018, 43(6): 28-31.

[61] 王登峰, 蔡珂芳, 张帅, 等. 基于激光拼焊技术的汽车 B 柱结构优化设计 [J]. 北京理工大学学报, 2018, 38(7): 691-697, 708.

WANG Dengfeng, CAI Kefang, ZHANG Shuai, et al. 
Structure design of automotive B-Pillar based on Tailor-Welded Blank technology[J]. Transactions of Beijing Institute of Technology，2018，38(7): 691-697, 708.

[62] SUN G Y, TAN D D, LÜ X J, et al. Multi-objective topology optimization of a vehicle door using multiple material tailor-welded blank (TWB) technology[J]. Advances in Engineering Software, 2018， 124: 1-9.

[63] 骆锐, 王艳, 吴沁. 汽车轻量化前沿制造技术的研究进 展 $[J]$. 制造技术与机床，2010(10)：142-145.

LUO Rui, WANG Yan, WU Qin. Research on front manufacturing technology of lightweight vehicle[J]. Manufacturing Technology \& Machine Tool, 2010(10): 142-145.

[64] 夏益新, 王娜, 陈新平, 等. 热冲压和液压成形技术在 宝钢汽车轻量化服务中的应用及发展趋势 [J]. 精密成 形工程, 2017, 9(6): 104-110.

XIA Yixin, WANG Na, CHEN Xinping, et al. Application and development trend of lightweight technology for vehicle with hot stamping and hydro-forming in Baosteel[J]. Journal of Netshape Forming Engineering, 2017, 9(6): 104-110

[65] 李洪波, 袁立峰. 液压成形在汽车车身成形上的应用分 析[J]. 模具制造, 2017，17(10): 21-24.

LI Hongbo, YUAN Lifeng. The application of hydro-forming technology in automobile body forming[J]. Die \& Mould Manufacture，2017，17(10): 21-24

[66] 蒋浩民, 陈新平, 范频, 等. 管件液压成形技术及其在 车身轻量化中的应用: 2007 中国钢铁年会 $[C] / /$ 中国四 川成都：冶金工业出版社，2007：339-344.

JIANG Haoming, CHEN Xinping, FAN Pin, et al. Tube hydro-forming technology and its application in lightweight of auto body[C]//2007 China Steel Annual Meeting, Chengdu, Sichuan, China: Metallurgical Industry Press, 2007: 339-344

[67] 朱梅云. 基于汽车轻量化的板材液压成形技术研究 [J]. 锻压装备与制造技术，2018，53(1)：88-90.

ZHU Meiyun. Study on technology of Sheet hydroforming based on automotive light-weight engineering $[\mathrm{J}]$. China Metalforming Equipment \& Manufacturing Technology, 2018，53(1): 88-90.

[68] 陈仙风, 徐小华, 苏海波. 液压成形汽车吸能盒研制关 键技术 $[J]$. 塑性工程学报, 2014, 21(2): 46-50. CHEN Xianfeng, XU Xiaohua, SU Haibo. Key technologies of hydro-forming automobile crash box[J].
Journal of Plasticity Engineering，2014，21(2): 46-50.

[69] 徐鸣涛, 王丽娟, 陈宗渝, 等. 基于管件液压成形工艺 的汽车吸能盒改进设计及成形分析 $[\mathrm{J}]$. 机械强度, 2017, 39(04): 864-869.

XU Mingtao, WANG Lijuan, CHEN Zongyu, et al. Automobile crash box improvement and forming analysis for tube hydro-forming[J]. Journal of Mechanical Strength, 2017, 39(4): 864-869.

[70] 罗爱华, SACHDEV A K, POWELL B R. 汽车轻量化 先进铸造技术 $[\mathrm{J}]$. 铸造， 2011，60(2): 113-119.

LUO Aihua, SACHDEV A K., POWELL B R. Advanced casting technologies for lightweight automotive applications[J]. Foundry, 2011, 60(2): 113-119.

[71] 吴伟斌, 廖劲威, 洪添胜, 等. 山地果园轮式运输机车 架结构分析与优化 [J]. 农业工程学报，2016，32(11): $39-47$.

WU Weibin, LIAO Jinwei, HONG Tiansheng, et al. Analysis and optimization of frame structure for wheeled transporter in hill orchard[J]. Transactions of the Chinese Society of Agricultural Engineering, 2016, 32(11): 39-47.

[72] 张娜娜, 赵匀, 刘宏新. 高速水稻插秧机车架的轻量化 设计[J]. 农业工程学报，2012，28(3): 55-59.

ZHANG Nana, ZHAO Yun, LIU Hongxin. Lightweight design of frame for self-propelled chassis rice transplanter[J]. Transactions of the Chinese Society of Agricultural Engineering, 2012，28(3): 55-59.

[73] 焦晨龙. 联合收割机机体的结构优化 $[\mathrm{D}]$. 天津: 河北工 业大学, 2015.

JIAO Chenlong. The optimization design of the body in combined harvester[D]. Tianjin: Hebei University of Technology, 2015.

[74] 藏世宇，吴崇友，伍扬华. 谷物联合收割机脱粒机机架 有限元分析及优化[J]. 江苏农业科学，2016，44(12): 347-349.

ZANG Shiyu, WU Chongyou, WU Yanghua. Finite element analysis and optimization of grain combine harvester thresher frame[J]. Jiangsu Agricultural Sciences, 2016, 44(12): 347-349.

[75] 韩红阳, 陈树人, 邵景世, 等. 机动式喷杆喷雾机机架 的轻量化设计[J]. 农业工程学报, 2013，29(3): 47-53, 293.

HAN Hongyang, CHEN Shuren, SHAO Jingshi, et al.Lightweight design of chassis frame for motor boom sprayer[J]. Transactions of the Chinese Society of Agricultural Engineering，2013，29(3): 47-53， 293. 
[76] 冯伟, 庞有伦, 李平, 等. 基于 ISIGHT 的小型收割机 机架优化设计研究 [J]. 西南农业学报, 2019, 32(1): 174-178.

FENG Wei, PANG Youlun, LI Ping, et al. Lightweight design for rack of small harvester based on ISIGHT[J]. Southwest China Journal of Agricultural Sciences, 2019, 32(1): 174-178.

[77] 周方思, 李立君, 欧阳益斌. 基于 ANSYS 的除草机车 架轻量化研究[J]. 林业工程学报, 2017, 2(6): 103-109. ZHOU Fangsi, LI Lijun, OUYANG Yibin. Lightweight research of mower frame based on ANSYS[J]. Journal of Forestry Engineering, 2017, 2(6): 103-109.

[78] 袁守利, 许炼. $3 \mathrm{WPZ}-500$ 自走式喷杆喷雾机机架的轻 量化设计[J]. 湖北农业科学, 2017, 56(7): 1359-1363. YUAN Shouli, XU Shuo. Lightweight design of frame for 3WPZ-500 self-propelled boom sprayer[J]. Hubei Agricultural Sciences, 2017, 56(7): 1359-1363.

[79] 陶雷, 王永健, 刘光远, 等. 秸秆打捆机机架轻量化设 计[J]. 中国农机化学报, 2017, 38(8): 26-33.

TAO Lei, WANG Yongjian, LIU Guangyuan, et al. Lightweight frame design of straw baler[J]. Journal of Chinese Agricultural Mechanization, 2017, 38(8): 26-33.

[80] 舒成松, 张大斌, 张云飞, 等. 烟秆拔秆破碎机机架轻 量化设计与动静态特性分析 [J]. 现代制造工程, 2019(6): 55-61, 67.

SHU Chengsong, ZHANG Dabin, ZHANG Yunfei, et al. Tobacco stalk pulling and shredding machine frame lightweight design and dynamic and static characteristic analysis[J]. Modern Manufacturing Engineering, 2019(6): $55-61,67$.

[81] 周林. 玉米收获机底盘车架强度分析及疲劳寿命研 究[D]. 北京: 中国农业机械化科学研究院, 2015 .

ZHOU Lin. Study on intensity analysis and fatigue life of corn combine chassis machine frame[D]. Beijing: Chinese Academy of Agricultural Mechanization Sciences, 2015.

[82] 鄢强, 邓祥丰, 陈代玉, 等. 谷物脱粒机机架的静力分 析与拓扑优化设计 [J]. 盐城工学院学报, 2019, 32(4): 12-17.

YAN Qiang, DENG Xiangfeng, CHEN Daiyu, et al. Static analysis and topology optimization design of grain thresher $\operatorname{rack}[\mathrm{J}]$. Journal of Yancheng Institute of Technology, 2019, 32(4): 12-17.

[83] 李耀明, 李有为, 徐立章, 等. 联合收获机割台机架结 构参数优化 [J]. 农业工程学报, 2014, 30(18): 30-37. LI Yaoming, LI Youwei, XU Lizhang, et al. Structural parameter optimization of combine harvester cutting bench[J]. Transactions of the Chinese Society of Agricultural Engineering, 2014, 30(18): 30-37.

[84] 生昕, 王杨, 金亦富, 等. 基于 ANSYS 的双轴旋耕机 机架轻量化设计 [J]. 农业装备技术, 2016, 42(4): 15-18. SHENG Xin, WANG Yang, JIN Yifu, et al. Lightweight design of dual-axis rotary tiller frame based on ANSYS[J]. Agricultural Equipment \& Technology, 2016, 42(4): $15-18$.

[85] 谢斌, 温昌凯，杨子涵，等. 基于实测载荷的蔬菜田间 动力机械车架结构优化[J]. 农业机械学报, 2018 , 49(S1): 463-469.

XIE Bin, WEN Changkai, YANG Zihan, et al. Structure optimization of frame for field vegetable power machinery based on measured load data[J]. Transactions of the Chinese Society for Agricultural Machinery, 2018, 49(S1): 463-469.

[86] 王利鹤, 赵永来, 崔红梅, 等. 基于 ANSYS Workbench 的深松机机架静力学分析及轻量化设计 $[\mathrm{J}]$. 重庆理工 大学学报, 2019, 33(2): 87-93.

WANG Lihe, ZHAO Yonglai, CUI Hongmei, et al. Static analysis and lightweight design of subsoiler frame based on ANSYS Workbench[J]. Journal of Chongqing University of Technology, 2019，33(2): 87-93.

[87] 刘炳昌. 设施蔬菜秸秆还田装备支架拓扑优化及分 析[J].南方农机，2020，51(1): 13， 29.

LIU Bingchang. Topology optimization and analysis of the equipment support for planting vegetable straw in the field[J]. China Southern Agricultural Machinery, 2020, 51(1): $13,29$.

[88］黄琼春, 李尚平, 李冰, 等. 小型甘蔗收获机台架结构 分析与轻量化设计 [J]. 农机化研究, 2018, 40(6): 29-32. HUANG Qiongchun, LI Shangping, LI Bing, et al. Structure analysis and lightweight design of the small sugarcane harvester stand[J]. Journal of Agricultural Mechanization Research，2018，40(6): 29-32.

[89] 龙超, 金秋谈, 陈哲吾, 等. 菱形收割机车架有限元分 析及优化 [J]. 机械科学与技术, 2014，33(1): 8-12. LONG Chao, JIN Qiutan, CHEN Zhewu, et al. Finite element analysis and optimization of rhombus harvester frame[J]. Mechanical Science and Technology for Aerospace Engineering, 2014，33(1): 8-12.

[90] 孙浭鹏, 林建, 刘灿灿, 等. 水稻收割机的割台轻量化 设计及经济性分析[J]. 江苏农业科学, 2019，47(4): 182-186. 
SUN Xiaopeng, LIN Jian, LIU Chanchan, et al. Lightweight design and economic analysis of header of rice harvester[J]. Jiangsu Agricultural Sciences, 2019, 47(4): 182-186.

[91] 徐敏雅, 张东风, 朱路生. 基于 ANSYS 的拖拉机下拉 杆拓扑优化设计及模态分析 [J]. 拖拉机与农用运输车, 2020，47(1): 32-35.

XU Minya, ZHANG Dongfeng, ZHU Lusheng. Topology optimization design and modal analysis of tractor lower link based on ANSYS[J]. Tractor \& Farm Transporter, 2020, 47(1): 32-35.

[92] 范思宇, 陆华忠, 丘广俊, 等. 果园电动履带运输车车 架轻量化设计[J]. 江苏农业科学, 2018, 46(6): 178-182. FAN Siyu, LU Zhonghua, QIU Guangjun, et al. Lightweight design of chassis frame for orchard electric tracked vehicle[J]. Jiangsu Agricultural Sciences, 2018, 46(6): 178-182.

[93] 胡建平, 黄否, 王留柱, 等. 基于参数敏感度分析的移 栽机提升支架轻量化设计 $[\mathrm{J}]$. 机械设计与制造, 2017(1): 38-42.

HU Jianping, HUANG Lei, WANG Liuzhu, et al. Research on lightweight design of transplanter lifting bracket based on parametric sensitivity analysis[J]. Machinery Design \& Manufacture, 2017(1): 38-42.

[94] 廖宇兰, 刘世豪, 孙佑攀, 等. 基于灵敏度分析的木薯 收获机机架结构优化设计[J]. 农业机械学报, 2013, 44(12): 56-61, 51.

LIAO Yulan, LIU Shihao, SUN Youpan, et al. Structural optimization for rack of cassava harvester based on sensitivity analysis[J]. Transactions of the Chinese Society for Agricultural Machinery, 2013，44(12): 56-61， 51.

[95] 沈伟, 廖敏, 王强, 等. 基于拓扑优化的变速箱壳体轻 量化设计[J]. 农机化研究，2018，40(4): 234-241.

SHEN Wei, LIAO Min, WANG Qiang, et al. Lightweight design of the transmission case based on topology optimization[J]. Journal of Agricultural Mechanization Research, 2018, 40(4): 234-241.

[96] 储婷婷, 朱德泉, 马锦, 等. 基于组合近似模型的分体 式插秧机秧箱轻量化设计 $[\mathrm{J}]$. 安徽农业大学学报, 2018, 45(5): 958-965.

CHU Tingting, ZHU Dequan, MA Jin, et al. Lightweight designs of split seedling box of rice transplanter based on ensemble approximate model[J]. Journal of Anhui Agricultural University, 2018, 45(5): 958-965.

[97] 胡奔, 廖敏, 李晓鹏, 等. 拖拉机前动力输出变速箱壳
体轻量化设计 [J]. 江苏农业科学, 2019，47(10)： 239-244.

HU Ben, LIAO Min, LI Xiaopeng, et al. Lightweight design of front power output gearbox housing for tractor[J]. Jiangsu Agricultural Sciences， 2019， 47(10): 239-244.

[98] 赵知辛, 薛旭东, 任江豪, 等. 小麦脱皮机转子的轻量 化设计 [J]. 食品与机械, 2020，36(6): 107-111.

ZHAO Zhixin, XUE Xiudong, REN Jianghao, et al. Lightweight design of wheat peeler rotor[J]. Food \& Machinery, 2020, 36(6): 107-111.

[99] 毛鹏军, 张亚龙, 丁月华, 等. 烟田辅助采收机车架的 轻量化设计 $[\mathrm{J}]$. 机械设计与制造, 2017(3)：89-92.

MAO Pengjun, ZHANG Yalong, DING Yuehua, et al. Lightweight design of the frame of tobacco field auxiliary harvesting machine[J]. Machinery Design \& Manufacture, 2017(3): 89-92.

[100] 潘群林. 丘陵山地拖拉机前转向驱动桥的轻量化研 究[D]. 成都：西华大学, 2019.

PAN Qunlin. Research on lightweight of front steering drive axle of tractor in hilly area[D]. Chengdu: Xihua University， 2019

[101] 吴锦标. 喷雾机喷杆轻量化设计及动态特性分析[D]. 武汉: 武汉理工大学, 2016.

WU Jinbiao. Lightweight design and dynamic characteristic analysis of sprayer boom[D]. Wuhan: Wuhan University of Technology, 2016.

[102] 张洪伟, 张以都, 王锡平, 等. 基于 ANSYS 参数化 建模的农用车车架优化设计 $[\mathrm{J}]$. 农业机械学报, 2007(3): 35-38.

ZHAG Hongwei, ZHANG, Yidu, WANG Xiping, et al. Optimization design of an agricultural vehicle frame based on ANSYS parametric modeling[D]. Transactions of the Chinese Society for Agricultural Machinery, 2007(3): 35-38

[103] YANG L H, ZHU C M, WANG Y J, et al. Lightweight design for the rear drive axle of the $10 \mathrm{t}$ parallel hybrid tractor[C]//Proceedings of 2015 International Conference on Advances in Management Science and Engineering (AMSE 2015): DEStech Publications, Jeep Island, Thailand, 2015: 114-119.

[104] 郑斌, 刘瑞祥, 孟建. 385 高压共轨柴油机连杆的有限 元分析与优化 [J]. 农机化研究, 2012, 34(11): 228-232. ZHEN Bin, LIU Ruixiang, MENG Jian. Finite element analysis and structural improvement of connecting rod of 
385 high pressure common diesel engine[J]. Journal of Agricultural Mechanization Research, 2012, 34(11): 228-232.

[105] 陈远帆, 李舜酩, 苏玉青. 拓扑优化与尺寸优化相结 合的割草车车架轻量化设计 $[\mathrm{J}]$. 重庆理工大学学报, 2017, 31(1): 28-35.

CHEN Yuanfan, LI Shunming, SU Yuqing.The light-weight design of the cutting grass car frame combined the topology optimization with the size optimization[J]. Journal of Chongqing University of Technology, 2017, 31(1): 28-35.

[106] 赵韩, 钱德猛. 基于 ANSYS 的汽车结构轻量化设计 [J]. 农业机械学报，2005(6): 12-15.

ZHAO Han, QIAN Demeng. Research on lightweight design of automobile structure based on ANSYS[J]. Transactions of the Chinese Society for Agricultural Machinery, 2005(6): 12-15.

[107] 马丽娜, 毛恩荣, 朱忠祥, 等. 轮式联合收获机转向 桥壳优化设计[J]. 农业机械学报, 2013, 44(S2): 283-287, 272.

MA Lina, MAO Enrong, ZHU Zhongxiang, et al. Optimized design of steering axle housing for wheeled combine harvester[J]. Transactions of the Chinese Society for Agricultural Machinery, 2013, 44(S2): 283-287, 272.

[108] 邓若玲, 王昱, 吕恩利, 等. 拖拉机液压机械无级变 速器箱体的轻量化设计 [J]. 农机化研究, 2019, 41(12): 258-263, 268.

DENG Ruoling, WANG Yu, LÜ Enli, et al. Lightweight design of hydro-mechanical continuously variable transmission box of the tractor[J]. Journal of Agricultural Mechanization Research, 2019, 41(12): 258-263, 268.

[109] 张毛宁, 张政武, 张东生. 基于 ANSYS 辊式磨粉机 机架静力学分析与结构优化 [J]. 粮食加工, 2019, 44(3): 47-50.

ZHANG Maoning, ZHANG Zhengwu, ZHANG Dongsheng. Static analysis and structure optimization of roller mill based on ANSYS[J]. Grain Processing, 2019, 44(3): $47-50$.

[110] 李怡, 徐中明, 张黎骅, 等. 大豆收获机车架的轻量 化设计[J]. 重庆大学学报, 2019, 42(10): 14-21.

LI Yi, XU Zhongming, ZHANG Lihua, et al. Light weight design of chassis frame for a soybean harvester[J]. Journal of Chongqing University, 2019, 42(10): 14-21.
[111］李海亮，梁琦，于珍珍，等. 宽幅播种机折叠式机架 的改进设计与优化 $[\mathrm{J}]$. 农机化研究, 2018, 40(6): 59-64.

LI Hailiang, LIANG Qi, YU Zhenzhen, et al. Improvement and optimization of wide planter folding-frame[J]. Journal of Agricultural Mechanization Research, 2018, 40(6): 59-64.

[112] 周成军, 刘富万, 周新年, 等. 穿轮配重轻型跑车及 其轻量化设计 $[\mathrm{J}]$. 福建农林大学学报, 2019, 48(6): 819-824.

ZHOU Chengjun, LIU Fuwan, ZHOU Xinnia, et al. Portable carriage with balanced weight and its lightweight design[J]. Journal of Fujian Agriculture and Forestry University, 2019, 48(6): 819-824.

[113] 赵映. 车载式钵苗栽种机底盘车架有限元分析及结构 优化[D]. 扬州: 扬州大学, 2012 .

ZHAO Ying. Finite element analysis and structure optimization of the chassis frame of the truck-mounted bowl seedling transplanter[D]. Yangzhou: Yangzhou University, 2012.

[114] 许学建. 履带式高地隙茶园管理机底盘行驶系统设 计[D]. 镇江: 江苏大学, 2016 .

XU Xuejian. Design of chassis traveling system of high-clearance tea garden management crawler[D]. Zhenjiang: Jiangsu University, 2016.

[115] 雷哓晖, 吕晓兰, 张美娜, 等. 背负式割草机刀盘轻 量化设计与试验[J]. 江西农业学报, 2018，30(1): 101-107.

LEI Xiaohui, LÜ Xiaolan, ZHANG Meina, et al. Lightweight design and test for cutting disc of shoulder-carrying Mower[J]. Acta Agriculturae Jiangxi, 2018, 30(1): 101-107.

[116] 李再参, 叶愈, 蒲宏毅, 等. 旋耕机机架结构优化设 计 [J]. 机电产品开发与创新, 2020, 33(6): 55-56, 63. LI Zaican, YE Yu, PU Hongyi, et al. Optimization design of rotary tiller frame structure[J]. Development \& Innovation of Machinery \& Electrical Products, 2020, 33(6): 55-56, 63.

[117] 张旻轩. 中小功率农林拖拉机两柱式 ROPS 的优化设 计与开发[D]. 上海: 上海交通大学, 2016 .

ZHANG Minxuan. Optimization design and development of two column ROPS for small and medium agriculture and forestry tractor[D]. Shanghai: Shanghai Jiao Tong University, 2016

[118] DU Y F, SONG Z H, LU F L, et al. Lightweight design 
of an axial flow roller based on sensitivity analysis[C]//

Proceedings of the 2015 International Conference on Materials, Environmental and Biological Engineering, Guilin, China: Atlantis Press, 2015: 998-1003.

[119] 李天箭, 丁晓红, 李郝林. 机床结构轻量化设计研究 进展 $[\mathrm{J}]$. 机械工程学报, 2020，56(21): 186-198.

LI Tianjian, DING Xiaohong, LI Haolin. Research progress on lightweight design of machine tool structure[J]. Journal of Mechanical Engineering, 2020, 56(21): 186-198.

[120] 晏梦雪, 田小永, 彭刚, 等. 轻质复合材料飞行器仪
器支架选择性激光烧结成形与性能研究 [J]. 机械工程 学报, 2019, 55(13): 144-150.

YAN Mengxue, TIAN Xiaoyong, PENG Gang, et al. Performance study of lightweight composites equipment section support fabricated by selective laser sintering $[\mathrm{J}]$. Journal of Mechanical Engineering, 2019, 55(13): 144-150.

作者简介: 才胜, 男, 1985 年出生, 博士, 讲师, 硕士研究生导师。主 要研究方向为轻量化技术、先进塑性加工工艺。

E-mail: sheng.cai@cau.edu.cn 